Published as: Nat Neurosci. 2013 September ; 16(9): 1315-1323.

\title{
Tuned Thalamic Excitation is Amplified by Visual Cortical Circuits
}

\author{
Anthony D. Lien and Massimo Scanziani \\ Neuroscience Graduate Program University of California San Diego, Howard Hughes Medical \\ Institute, Center for Neural Circuits and Behavior, Neurobiology Section and Department of \\ Neuroscience, La Jolla, California 92093-0634, USA
}

\begin{abstract}
Cortical neurons in thalamic recipient layers receive excitation from the thalamus and the cortex. The relative contribution of these two sources of excitation to sensory tuning is poorly understood. Here we optogenetically silence the visual cortex of mice to isolate thalamic excitation onto layer 4 neurons during visual stimulation. Thalamic excitation contributes to a third of total excitation and is organized in spatially offset, yet overlapping ON and OFF receptive fields. This receptive field structure predicts the orientation tuning of thalamic excitation. Finally, thalamic and total excitation are similarly tuned to orientation and direction, and have the same temporal phase relationship to the visual stimulus. Our results indicate that tuning of thalamic excitation is unlikely to be imparted by direction or orientation selective thalamic neurons and that a principal role of cortical circuits is to amplify tuned thalamic excitation.
\end{abstract}

\section{Introduction}

Synaptic excitation plays an important role in shaping the sensory tuning properties of cortical neurons ${ }^{1-6}$. When located in thalamic recipient layers these neurons receive synaptic excitation from two major sources: thalamic and cortical neurons. The relative contribution of these two sources to the tuning of cortical neurons is debated.

In the visual system both thalamic and cortical neurons respond robustly to visual stimuli consisting of alternating bars of high and low luminance (gratings) that move across the visual field causing cyclical changes in luminance at each location in visual space. Due to the spatial arrangement of their receptive fields, many neurons spike preferentially at specific temporal phases of the grating cycle and are thus modulated at the temporal frequency of the grating. In addition to phase modulation, neurons may exhibit sensitivity to the orientation of the grating, responding with more spikes at certain orientations as compared to others.

\footnotetext{
Users may view, print, copy, download and text and data- mine the content in such documents, for the purposes of academic research, subject always to the full Conditions of use: http://www.nature.com/authors/editorial_policies/license.html\#terms

Author Contributions

A.D.L. and M.S. designed the study. A.D.L. conducted all experiments and analysis. A.D.L. and M.S. wrote the paper.

Competing Financial Interests

The authors declare no competing financial interests.
} 
In their classic feedforward model, Hubel and $\mathrm{Wiesel}^{7}$ proposed that thalamic excitation of a cortical neuron could be orientation selective via the convergence of multiple untuned thalamic inputs with properly aligned spatial receptive fields. There is evidence for such receptive field arrangements among the population of thalamic neurons projecting to the same orientation domain of visual cortex in ferrets ${ }^{8}$ and cats ${ }^{9}$. Additionally, cortical neurons whose receptive field structure overlaps with the receptive field structure of individual thalamic neurons are indeed more likely to receive input from these neurons as compared to neurons with non matching receptive fields ${ }^{10,11}$. Yet, the tuning properties of synaptic excitatory currents resulting from the convergence of a set of thalamic inputs onto individual cortical neurons are still unclear.

Furthermore, the impact of cortical excitation in shaping the total excitation received by visual cortical neurons is largely unknown. In mouse visual cortex, L2/3 neurons with similar orientation preference are more likely to be connected ${ }^{12}$ and long-range intracortical projections in the visual cortex of other species preferentially connect domains sharing similar orientation preference ${ }^{13-15}$, suggesting that cortical excitation might contribute to the orientation tuning of total excitation.

A pivotal study combining intracellular recording in cat visual cortex with cooling-mediated cortical silencing isolated thalamic excitation onto cortical neurons and demonstrated its tuning to orientation ${ }^{16}$. However, because the receptive field structure of thalamic excitation, i.e. the spatial organization of $\mathrm{ON}$ and $\mathrm{OFF}$ receptive fields, was not assessed in these studies, the mechanisms underlying the observed orientation tuning could not be addressed. Furthermore, the relationship between thalamic and total excitation could not be established because synaptic activity recorded without cortical cooling reflected the combined effects of excitation and inhibition.

We have used an optogenetic strategy for cortical silencing in which we photostimulate parvalbumin-expressing (PV) cortical GABAergic interneurons ${ }^{17}$ expressing the light-gated cation channel channelrhodopsin $2(\mathrm{ChR} 2)^{18,19}$. By harnessing the dense and divergent inhibitory axons of PV cells we can completely silence the cortex for prolonged periods of time without affecting the dynamics of transmitter release from thalamic terminals.

Using this approach we reveal how the ON and OFF receptive field structure of thalamic excitation onto layer 4 cortical neurons can account for its orientation tuning properties. Consistent with this hypothesis, we show that in response to drifting gratings, the phase modulation of thalamic excitation was tuned to orientation and direction, but the integral of thalamic excitatory current across the stimulus duration was untuned. Finally we demonstrate that the orientation and direction tuning as well as the phase of cortical excitation onto layer 4 neurons matches that of their thalamic excitation. These findings indicate that the phase and orientation preference of thalamic excitation are reinforced by cortical excitation and are consistent with the possibility that orientation and direction tuning of thalamic excitation is generated by individually untuned thalamic inputs. 


\section{Results}

\section{Isolating thalamic excitation}

To isolate the thalamic component of visually evoked synaptic excitation in layer 4 (L4) neurons in vivo, we optogenetically silenced the mouse visual cortex (Fig. 1a). The cortex was silenced by photostimulation of inhibitory parvalbumin-expressing (PV) GABAergic cortical interneurons that conditionally expressed the light-sensitive cation channel channelrhodopsin 2 (ChR2). We evaluated the robustness of cortical silencing by recording, in loose-patch configuration, the spiking responses of individual V1 neurons to visual stimuli consisting of drifting gratings with and without PV cell photostimulation on interleaved trials (Fig. 1b). Illumination of the cortical surface with a blue $(470 \mathrm{~nm})$ lightemitting diode (LED) for $2.6 \mathrm{~s}$ completely silenced both the spontaneous and visual stimulus-evoked spiking of all recorded neurons throughout the entire illumination period (Fig. 1b, n=14 cells, 2 mice). Experiments in which the local field potential (LFP) was simultaneously recorded with a second electrode while the LED intensity was varied to decrease cortical activity to different levels revealed a strong correlation between the reduction in visually evoked firing rate and LFP amplitude (11 cells, 4 mice; Supplementary fig. 2). Complete cortical silencing reduced the amplitude of the visually-evoked LFP by $\sim 80 \%$ (Supplementary fig. 2). Thus, the LFP is a reliable indicator of cortical activity and was routinely monitored in subsequent experiments to verify cortical silencing (see Methods). These data indicate that complete cortical silencing can be achieved by photostimulating ChR2-expressing PV cells, consistent with previous reports from our $1 \mathrm{ab}{ }^{17,20}$.

We recorded from L4 neurons (average depth: $386 \pm 7 \mu \mathrm{m}$, restricted to 300-550 $\mu \mathrm{m}, \mathrm{n}=49$ cells, 40 mice; Supplementary fig. 1) in the whole-cell voltage clamp configuration and used visual stimulation to evoke excitatory postsynaptic currents (EPSCs) with and without cortical silencing on interleaved trials. Visual stimuli consisted of either an $8 \times 8$ grid of individually flashed black or white squares (Fig. 1c) or full-field gratings drifting in 12 different directions (Fig. 1d). Cortical silencing reduced the total excitatory postsynaptic charge (Q: the time integral of the EPSC) evoked by the flashed square of optimal location and luminance (i.e. the luminance and location that evoked the largest $\mathrm{Q}$ in control conditions) by $68 \pm 3 \%$ (Fig. 1c; control: mean EPSC: $-60 \pm 9$ pA, Q: $15 \pm 2$ pC; cortical silencing: mean EPSC: $-17 \pm 2 \mathrm{pA}, \mathrm{Q}: 4.4 \pm 0.6 \mathrm{pC}, \mathrm{n}=18$ cells, 16 mice) and gratings drifting in the preferred direction (i.e. the direction that evoked the largest $\mathrm{Q}$ in control conditions) by $64 \pm 2 \%$ (Fig. 1d; control: mean EPSC: $-143 \pm 10$ pA, Q: $239 \pm 17$ pC; cortical silencing: mean EPSC: $-46 \pm 3$ pA, Q: $78 \pm 4$ pC, $n=42$ cells, 33 mice). Thus, because in the absence of cortical activity the principal remaining synaptic input to cortical neurons comes from the thalamus, these results indicate that the thalamic component of excitation contributes to $32 \pm 3 \%$ and $36 \pm 2 \%$ of the total excitation evoked by flashed squares and drifting grating stimuli, respectively (Fig. 1c-d). This estimate is likely to be an upper bound as cortical silencing under these conditions has been shown to increase the activity of thalamic relay neurons due to the elimination of the cortico-thalamic feedback loop ${ }^{17}$ (Supplementary Fig. 4g). In contrast, the release of GABA (by photostimulated PV cells) did not, per se, substantially affect the estimate of the thalamic component: The 
decrease in the membrane resistance of the recorded neuron via the activation of $\mathrm{GABA}_{\mathrm{A}}$ receptors was only $32 \pm 3 \%$ ( $\mathrm{n}=15$ cells, 11 mice) and a decrease in transmitter release via activation of presynaptic $\mathrm{GABA}_{\mathrm{B}}$ receptors on thalamic terminals could be ruled out (Supplementary fig. 3). Below, we refer to the EPSC and Q recorded during control trials as EPSC $_{\text {Tot }}$ and $\mathrm{Q}_{\text {Tot }}$, respectively, and to the EPSC and Q recorded during cortical silencing trials as $\mathrm{EPSC}_{\text {Thal }}$ and $\mathrm{Q}_{\text {Thal }}$, respectively.

\section{Receptive field structure of thalamic excitation}

To determine the receptive field structure of thalamic excitation onto individual layer 4 cortical neurons we silenced the cortex while presenting the $8 \times 8$ grid of flashed black or white squares (see above) and recorded $\mathrm{EPSC}_{\text {Thal }}$ evoked at each grid location. (Fig. 2a).

We generated separate receptive field maps of thalamic excitation for black (OFF) and white (ON) stimuli based on the value of $\mathrm{Q}_{\mathrm{Thal}}$ for a $100 \mathrm{~ms}$ window following stimulus onset for each grid location (Fig. 2a heat map). Individual OFF and ON subfields were defined as spatially continuous regions of the receptive field map exceeding the background (Fig. 2b, see methods).

In 17 out of 18 cells, at least one subfield could be identified. Out of these cells, 13 had both a single ON and single OFF subfield while the remaining 4 had a single OFF subfield. We quantified the spatial properties of the receptive field in those 13 cells (12 mice) that exhibited both ON and OFF subfields.

The average area of a subfield for $\mathrm{EPSC}_{\text {Thal }}$ was $242 \pm 21 \mathrm{deg}^{2}$ ( $\mathrm{n}=26$ subfields; Fig. 2f). However, the ON and OFF subfields for individual cells were highly overlapping in space with an average overlap area of $167 \pm 26 \mathrm{deg}^{2}$ ( $\mathrm{n}=13$ cells; Fig. 2f, overlap area). To quantify the overlap of the ON and OFF subfields we used the overlap index, defined as the fraction of the smaller subfield that was overlapped by the larger subfield. The average overlap index was $0.79 \pm 0.07$ ( $n=13$ cells; Fig. 2f, overlap index).

Despite the high degree of overlap, many neurons exhibited a spatial offset between the peaks of the ON and OFF subfields. The average distance between ON and OFF peaks was $5.1 \pm 0.5$ degrees $(\mathrm{n}=13$ cells; Fig. $2 \mathrm{~g})$.

To ensure that the separation between the peaks of ON and OFF subfields that we measured was not due to noise in the estimate of peak location, we simulated the probability that a pair of identical subfields would exhibit a similar degree of peak separation given the variability of responses measured in pixels outside of the receptive field (see Methods). The probability that the measured separation between the peaks of ON and OFF subfields was less than the separation between identical subfields was below 0.05 for $12 / 13$ cells and below 0.005 for $9 / 13$ cells.

We measured the elongation of the subfields by comparing the width of each subfield along the axis connecting the peaks of the ON and OFF subfields (ON-OFF axis) with the width along the orthogonal axis (Fig. 2h). The subfield widths along these two axes were similar $(\mathrm{ON} / \mathrm{OFF}$ axis width $=10.6 \pm 0.7$ degrees, orthogonal axis width $=11.4 \pm 0.7$ degrees, $\mathrm{p}=$ 0.3 , not statistically significant; $\mathrm{n}=26$ subfields). The aspect ratio, the width along the 
orthogonal axis divided by the width along the ON-OFF axis, was close to 1 (aspect ratio $=$ $1.15 \pm 0.07 ; \mathrm{n}=26$ subfields).

Thus the spatial receptive field of the thalamic excitatory input onto an individual cortical neuron consists of roughly symmetric spatially overlapping ON and OFF subfields whose peaks are offset by about 5 degrees in visual space.

\section{Orientation tuning of thalamic excitation}

The separation between the peaks of the ON and OFF EPSC $\mathrm{C}_{\text {Thal }}$ subfields suggests that in response to drifting gratings, $\mathrm{EPSC}_{\text {Thal }}$ could, in principle, be orientation tuned without the average firing rate (averaged over the stimulus duration) of thalamic neurons contributing to EPSC $_{\text {Thal }}$ being itself orientation tuned. If the drifting grating is presented at an orientation where the bars of the grating are perpendicular to the axis connecting the peaks of $\mathrm{ON}$ and OFF thalamic subfields, it should produce simultaneous activation of ON and OFF thalamic subfields at the same temporal phase of the grating cycle (Fig. 4h, left). The resulting $\mathrm{EPSC}_{\text {Thal }}$ will fluctuate in amplitude at the same temporal frequency of the grating (F1 modulation) and be maximal when the two thalamic subfields are activated together. The difference between the peak and the trough of this $\mathrm{EPSC}_{\text {Thal }}$ fluctuation is the $\mathrm{F} 1$ amplitude $\left(\mathrm{F} 1_{\text {Thal }}\right)$. In contrast, orthogonal gratings would activate the subfields sequentially at different temporal phases producing a smaller $\mathrm{F} 1_{\text {Thal }}$ (Fig. 4h, right). In other words, $\mathrm{F} 1_{\text {Thal }}$ should be tuned to grating orientation.

Furthermore, if the average firing rate of thalamic neurons that contribute to $\mathrm{EPSC}_{\mathrm{Thal}}$ is not orientation selective, the number of action potentials generated in these neurons over the entire stimulus duration should be the same regardless of the orientation of the grating. Hence, the integral of $\mathrm{EPSC}_{\text {Thal }}$ over the stimulus duration, $\mathrm{Q}_{\text {Thal }}$, should be the same at each orientation and so $\mathrm{Q}_{\mathrm{Thal}}$ should be untuned to grating orientation.

In contrast, if a cortical neuron receives input from a population of thalamic neurons whose average firing rate is orientation tuned and share similar orientation preference, then $\mathrm{Q}_{\text {Thal }}$ should be tuned to grating orientation. The tuning of $\mathrm{F} 1_{\text {Thal }}$ in this case would depend on the degree to which the thalamic inputs fire at the same phase of the grating cycle.

Thus, to examine the orientation tuning of thalamic excitation we extracted two values from $\mathrm{EPSC}_{\text {Thal }}$ recorded during drifting gratings: $\mathrm{F} 1_{\text {Thal }}$ and $\mathrm{Q}_{\text {Thal }}$ (Fig. 3a, right). Note that $\mathrm{Q}$ is equivalent to the traditionally used F0, which is the average value of the response across the entire stimulus duration.

$\mathrm{Q}_{\text {Thal }}$ was remarkably similar across all grating orientations, resulting in a flat tuning curve and a low value of the orientation and direction selectivity index (OSI $=0.026 \pm 0.003$; DSI $=0.083 \pm 0.009 ; \mathrm{n}=42$ cells; Fig. $3 \mathrm{~b}-\mathrm{e}$ ). In contrast to $\mathrm{Q}_{\text {Thal }}, \mathrm{F} 1_{\text {Thal }}$ was clearly selective for orientation and direction (OSI F1 $1_{\text {Thal }}=0.23 \pm 0.017$, significantly different than OSI $\mathrm{Q}_{\text {Thal }}, \mathrm{p}=5.8 \mathrm{e}-15 ; \mathrm{DSI} 1_{\text {Thal }}=0.28 \pm 0.03$, significantly different than DSI $\mathrm{Q}_{\text {Thal, }} \mathrm{p}=$ $4.4 \mathrm{e}-9 ; \mathrm{n}=42$ cells; Fig. $3 \mathrm{~b}-\mathrm{e})$. Thus orientation and direction tuning is present in $\mathrm{F}_{\text {Thal }}$, but not in $\mathrm{Q}_{\text {Thal }}$. 
Consistent with the absence of $\mathrm{Q}_{\text {Thal }}$ tuning, recordings from single units in the dorsal lateral geniculate nucleus (dLGN) during cortical silencing indicated that the average firing rate of individual thalamic neurons was poorly tuned to orientation (OSI $=0.067 \pm 0.01 \mathrm{n}=11$ units; Supplementary Fig. 4e). Finally, the F1 modulation of the firing of thalamic neurons was also poorly tuned to orientation, significantly less than the $\mathrm{F} 1_{\text {Thal }}$ recorded in cortical neurons (OSI $=0.11 \pm 0.021$; unpaired t-test, $\mathrm{p}=0.0002 ; \mathrm{n}=11$; Supplementary Fig. $4 \mathrm{~h})$. In fact, while in $55 \%$ of the cortical neurons the OSI of $\mathrm{F} 1_{\text {Thal }}$ was larger than 0.2 , this value was reached by only $9 \%$ of the thalamic units recorded during cortical silencing (Supplementary Fig. 4h).

\section{Receptive field structure predicts preferred orientation of thalamic excitation}

If the separation between the peaks of the ON and OFF EPSC $\mathrm{Chal}_{\text {Tha }}$ subfields is a major determinant of the observed orientation tuning of $\mathrm{F} 1_{\text {Thal }}$, then the relative position of the $\mathrm{ON}$ and OFF peaks should predict the preferred orientation of $\mathrm{F}_{\text {Thal }}$.

To test this, we obtained both receptive field maps (Fig. 4a-b) and drifting grating orientation tuning curves (Fig. $4 \mathrm{c}-\mathrm{d}$ ) of thalamic excitation in the same neuron. The predicted preferred orientation derived from receptive field maps $\left(\mathrm{RF}_{\text {Pref }}\right)$ was determined by the angle of the line connecting the peaks of the ON and OFF subfields (Fig. 4b). The relationship between $\mathrm{RF}_{\text {Pref }}$ and the actual preferred orientation of $\mathrm{F} 1_{\text {Thal }}$ (Grating Pref $_{\text {) }}$ can be seen by overlaying vectors indicating $\mathrm{RF}_{\text {Pref }}$ (black dashed line) and Grating ${ }_{\text {Pref }}$ (blue line) on the polar orientation tuning curve (Fig. $4 d-e$ ). $R_{\text {Pref }}$ and Grating ${ }_{\text {Pref }}$ were remarkably similar, differing by less than 30 degrees in 7 out of 8 cells (mean difference in preferred orientation: $19 \pm 7$ degrees; Fig. $4 \mathrm{f}-\mathrm{g}$ ). Thus the separation of ON and OFF subfields is likely to be a key determinant of the preferred orientation of $\mathrm{F} 1_{\text {Thal }}$

\section{Tuning of non-thalamic excitatory charge}

Is $\mathrm{Q}_{\mathrm{Tot}}$, that is the charge recorded in the absence of cortical silencing, also untuned to orientation and direction like $\mathrm{Q}_{\text {Thal }}$ ? Comparison of the tuning curves for $\mathrm{Q}_{\mathrm{Tot}}$ and $\mathrm{Q}_{\mathrm{Thal}}$ revealed that $\mathrm{Q}_{\text {Tot }}$ was in fact significantly more selective for orientation and direction than $\mathrm{Q}_{\text {Thal }}\left(\mathrm{OSI} \mathrm{Q}_{\mathrm{Tot}}=0.049 \pm 0.005, \mathrm{p}=1.7 \mathrm{e}-4 ; \mathrm{DSI} \mathrm{Q}_{\text {Tot }}=0.12 \pm 0.01, \mathrm{p}=0.007 ; \mathrm{n}=42\right.$ cells; Fig. 5b-e). This suggests that under control conditions, when cortical activity is intact, the excitatory charge that is not of thalamic origin is, in contrast to $\mathrm{Q}_{\mathrm{Thal}}$, orientation and direction selective. We isolated this component of excitation by subtracting $\mathrm{EPSC}_{\text {Thal }}$ from $\mathrm{EPSC}_{\text {Tot }}\left(\mathrm{EPSC}_{\mathrm{Sub}}=\mathrm{EPSC}_{\mathrm{Tot}}-\mathrm{EPSC}_{\text {Thal }}\right.$; Fig. 5a). Given the complete silencing of cortical activity upon PV cell photostimulation, $\mathrm{EPSC}_{\text {sub }}$ must, in large part, reflect cortical excitation. $\mathrm{Q}_{\mathrm{Sub}}$ (the charge integral of $\mathrm{EPSC}_{\mathrm{Sub}}$ ) was even more orientation and direction selective than $\mathrm{Q}_{\text {Tot }}\left(\mathrm{OSI} \mathrm{Q}_{\mathrm{Sub}}=0.083 \pm 0.009 ; \mathrm{p}=1.4 \mathrm{e}-8\right.$; DSI $\mathrm{Q}_{\mathrm{Sub}}=0.18 \pm 0.017 ; \mathrm{p}=$ $2 \mathrm{e}-9 ; \mathrm{n}=42$ cells) and more selective than $\mathrm{Q}_{\text {Thal }}$ (OSI $\mathrm{p}=6 \mathrm{e}-7$; DSI $\mathrm{p}=3.9 \mathrm{e}-6 ; \mathrm{n}=42$ cells; Fig. 5f-i). Thus, in contrast to $\mathrm{Q}_{\mathrm{Thal}}$, both $\mathrm{Q}_{\mathrm{Tot}}$ and $\mathrm{Q}_{\mathrm{Sub}}$ are tuned to orientation and direction.

\section{Tuning of non-thalamic F1 modulation}

Similar to $\mathrm{EPSC}_{\text {Thal }}, \mathrm{F} 1$ modulation of $\mathrm{EPSC}_{\mathrm{Sub}}$ was evident particularly at certain orientations (Fig. 6a). We quantified the orientation and direction selectivity of the F1 
modulation of $\mathrm{EPSC}_{\mathrm{Sub}}\left(\mathrm{F}_{\text {Sub }}\right)$ and compared it to the tuning of $\mathrm{Q}_{\text {Sub }}$ (Fig. 6b-g). $\mathrm{F} 1_{\text {Sub }}$ was significantly more selective for orientation and direction than $\mathrm{Q}_{\mathrm{Sub}}\left(\mathrm{OSI}\right.$ of $\mathrm{F} 1_{\mathrm{Sub}}$ : 0.24 $\pm 0.02, p=1.2 \mathrm{e}-10 ;$ DSI of $F 1_{\text {Sub }}=0.38 \pm 0.04 ; p=4.4 \mathrm{e}-6 ; n=42$ cells; Fig. $6 \mathrm{~d}$ and $\mathrm{f}$ ). Importantly, however, $\mathrm{F}_{\mathrm{Sub}}$ and $\mathrm{Q}_{\mathrm{Sub}}$ were tuned to the same orientation (mean difference between $\mathrm{F} 1_{\text {Sub }}$ and $\mathrm{Q}_{\text {Sub }}$ preferred orientation: $20 \pm 3$ degrees, $\mathrm{n}=42$ cells; Fig. 6e). Furthermore $\mathrm{F}_{\text {Sub }}$ and $\mathrm{Q}_{\text {Sub }}$ were also tuned to similar directions (Fig. 6g). Thus $\mathrm{F} 1_{\text {Sub }}$ exhibits orientation and direction selectivity that is co-tuned with $\mathrm{Q}_{\mathrm{Sub}}$.

\section{Co-tuning of thalamic and non-thalamic excitation}

Both $\mathrm{F} 1_{\text {Thal }}$ and $\mathrm{F} 1_{\text {Sub }}$ are orientation selective. Are these two sources of excitation tuned to the same or different orientations? The preferred orientations of $\mathrm{F} 1_{\text {Thal }}$ and $\mathrm{F} 1_{\text {Sub }}$ differed by only $20 \pm 3$ degrees on average ( $\mathrm{n}=42$ cells; Fig. $7 \mathrm{~d}$, top) and the OSI of $\mathrm{F} 1_{\text {Thal }}$ and $\mathrm{F} 1_{\text {Sub }}$ were not significantly different (Thal: $0.22+/-0.015$, Sub: $0.24 \pm 0.02, p=0.63 ; n=42$ cells; Fig. 7d, bottom). In addition $\mathrm{F} 1_{\text {Thal }}$ and $\mathrm{F} 1_{\text {Sub }}$ were also tuned to similar directions (Fig. 7e, top) yet DSI of $F 1_{\text {Thal }}$ was significantly smaller than DSI of F1 Sub (Thal: $0.23 \pm$ 0.017, Sub: $0.37 \pm 0.04, P=0.03 ; n=42$ cells; Fig. 7e, bottom). Finally, we determined the phase difference between $\mathrm{F}_{1}$ Thal and $\mathrm{F} 1_{\text {Sub }}$ at the preferred direction of thalamic excitation. We found that $\mathrm{F} 1_{\text {Thal }}$ and $\mathrm{F} 1_{\text {Sub }}$ were almost in phase (Fig. $7 \mathrm{f}$ ), with $\mathrm{EPSC}_{\text {Thal }}$ preceding EPSC $_{\text {Sub }}$, on average by $30.3 \pm 5.5$ degrees or $42 \pm 8 \mathrm{~ms}$ given the temporal frequency of our stimulus ( $\mathrm{n}=42$ cells; Fig. $7 \mathrm{~g}$ ). Thus $\mathrm{F} 1_{\text {Thal }}$ and $\mathrm{F} 1_{\text {Sub }}$ are co-tuned to orientation and have similar temporal phase.

\section{Discussion}

Here we describe the tuning properties of the thalamic and total excitation received by single neurons in L4 of mouse visual cortex using intracellular voltage clamp recordings and optogenetic cortical silencing. Our recordings reveal that thalamic excitation is organized into spatially offset yet highly overlapping ON and OFF receptive fields. The relative position of the $\mathrm{ON}$ and OFF subfields is predictive of the orientation preference of thalamic excitation in response to drifting gratings. This orientation selectivity is observed in the F1 modulation of thalamic excitation $\left(\mathrm{F} 1_{\text {Thal }}\right)$, but not in the thalamic excitatory charge $\left(\mathrm{Q}_{\text {Thal }}\right)$ demonstrating that it does not arise from the convergence of thalamic neurons that are themselves orientation selective. Finally, because the thalamic and cortical contributions to total excitation are tuned to the same orientation and share similar temporal phase, our data demonstrate a key function of visual cortex in amplifying tuned thalamic excitation.

We find that the thalamus contributes on average approximately $30 \%$ of the excitatory charge to a cortical neuron. This is consistent with previous studies using intracellular recording and cortical silencing in the cat visual cortex $\left(37 \% 16\right.$ and $\left.46 \%{ }^{21}\right)$ yet less than what was reported in rat auditory cortex $\left(61 \%^{6}\right)$. Our estimation of the thalamic contribution is likely to be an upper bound due to the facilitation of thalamic responses to visual stimuli upon disruption of corticothalamic feedback with cortical silencing ${ }^{17}$ (Supplementary Fig. 4). Additionally, the potential underestimation of total excitation (recorded without cortical silencing) in case of improper voltage clamp may further increase our estimate of the thalamic component. Finally, the two potential sources of under-estimation of the thalamic 
component, leakier postsynaptic membrane during cortical silencing and inhibition of transmitter release from thalamic afferents play only a minor role. PV cell photostimulation decreased input resistance of the recorded cells by only 30\%; given the perisomatic distribution of PV cell synapses, the conductance underlying this decrease in resistance is likely to be properly voltage clamped. Furthermore, the potential activation of presynaptic $\mathrm{GABA}_{\mathrm{B}}$ receptors on thalamic terminals via GABA release from photostimulated PV cells did not affect transmitter release (Supplementary Fig. 3).

We provide the first description, to our knowledge, of the ON and OFF receptive fields of thalamic excitation onto visual cortical neurons. The ON and OFF subfields of a single cell are highly overlapping, have little elongation, but exhibit spatially offset peaks. The average subfield width is 10 degrees, which is similar to the average width of the center receptive field of a neuron in the dorsal lateral geniculate nucleus (dLGN) of the mouse thalamus ( 9.8 degrees $^{22}$ and 11 degrees $^{23}$ ). The receptive fields of thalamic excitation reported here are smaller than previously reported receptive fields of total synaptic excitation ${ }^{3}$. A possible reason for this difference is that in our study intracortical connections are functionally eliminated via cortical silencing. If intracortical connections originate in part from cortical neurons whose receptive field is outside of or larger than the thalamic RF of the recorded neuron then intracortical connections would contribute to broadening the RF size.

We believe that the spatial offset of the ON and OFF subfields forms the basis for orientation selectivity of thalamic excitation. When the drifting grating is perpendicular to the axis connecting the ON and OFF peaks, it will simultaneously activate both ON and OFF subfields once per grating cycle resulting in strong F1 modulation (Fig. 4h, left). At the orthogonal orientation, the $\mathrm{ON}$ and OFF peaks will be activated sequentially at opposite phases of the grating cycle, resulting in weak F1 modulation (Fig. 4h, right). We find that $\mathrm{F} 1_{\text {Thal }}$ is indeed well tuned for orientation, consistent with observations in cat visual cortex $^{16}$.

In contrast, the excitatory charge over the stimulus period, $\mathrm{Q}_{\text {Thal }}$, is poorly tuned, consistent with the Hubel and Wiesel feedforward model in which the receptive field structure of thalamic inputs rather than the tuning of individual thalamic neurons forms the basis of orientation selectivity in cortical neurons ${ }^{7}$. Indeed if the firing rate of thalamic neurons was tuned for orientation, the resulting $\mathrm{Q}_{\text {Thal }}$ should also be tuned, which is not what we observe. Thalamic neurons that are tuned to direction and orientation have been recently described in the mouse thalamus ${ }^{22,24}$. However, and consistent with our own dLGN recordings (Supplementary Fig. 4), they constitute a small fraction (11\%) of the neurons in mouse dLGN, which appears to be dominated by cells with poor direction and orientation selectivity ${ }^{22}$. Hypothetically, the F1 modulation but not the firing rate of thalamic neurons could be tuned for orientation. This could lead to the observed $\mathrm{F} 1_{\text {Thal }}$ tuning and concomitant lack of $\mathrm{Q}_{\text {thal }}$ tuning. This is, however, unlikely because the F1 tuning of dLGN neurons was poor (Supplementary Fig. 4). Furthermore, the few dLGN neurons with tuned F1 modulation also appear to exhibit similar tuning of their firing rates ${ }^{24}$. Nevertheless it is possible that orientation and/or direction selective dLGN neurons may contribute to the tuning properties of cortical neurons outside of L4, the layer that we targeted in this study. 
It has been established in several species that the structure of the spike receptive fields of cortical simple cells also predicts the preferred orientation of spiking responses ${ }^{4,25}$. Furthermore, the receptive field structure of populations of thalamic inputs in cat visual cortex predicts the preferred orientation of the target domain ${ }^{9}$. Nevertheless our results are the first to demonstrate that the close relationship between receptive field structure and preferred orientation previously observed in the spiking output is already present in thalamic excitation onto single cortical neurons.

It is arguable that cortical silencing may affect orientation and direction tuning of thalamic neurons via the disruption of corticothalamic feedback. However, our recordings of dLGN units demonstrate little change to their tuning properties during cortical silencing (Supplementary Figure 4). This is consistent with previous experiments addressing the impact of cortex on thalamic tuning properties which find effects on overall gain and spatial frequency tuning ${ }^{26}$, but do not report changes in orientation or direction tuning.

While some functional properties of visual cortex such as spike rate ${ }^{27}$, surround suppression $^{28}$, and inhibition ${ }^{29}$ are influenced by anesthesia, other properties such as orientation tuning ${ }^{27}$ remain relatively unaffected. Thus we believe that the mechanisms underlying orientation tuning revealed here in anesthetized animals are likely to apply under awake conditions.

We isolated the excitatory component that is not of thalamic origin $\left(\mathrm{EPSC}_{\mathrm{Sub}}\right)$ by subtracting the thalamic excitation $\left(\mathrm{EPSC}_{\mathrm{Thal}}\right)$ from the total excitation $\left(\mathrm{EPSC}_{\mathrm{Tot}}\right)$. Because of the complete silencing of the cortex upon photostimulation of PV cells, $\mathrm{EPSC}_{\mathrm{Sub}}$ is likely to mainly reflect excitation mediated by cortical neurons. Furthermore because of our overestimate of thalamic excitation during cortical silencing (discussed above) $\mathrm{EPSC}_{\mathrm{Sub}}$ is likely to be an underestimate of cortical excitation. Could the observed tuning of $\mathrm{EPSC}_{\mathrm{Sub}}$ simply result from the subtraction of an overestimated thalamic excitation from $\mathrm{EPSC}_{\mathrm{Tot}}$ ? If so this would bias the tuning properties of $\mathrm{EPSC}_{\mathrm{Sub}}$ to be actually anti-correlated with

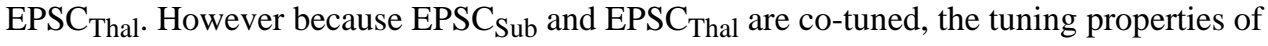
EPSC $_{\text {Sub }}$ are unlikely to be due to subtraction artifacts. In fact, the subtraction of an overestimated EPSC $_{\text {Thal }}$ likely underestimates the degree of co-tuning of thalamic and cortical excitation.

While EPSC $_{\text {Sub }}$ must largely reflect the synaptic excitation contributed by cortical neurons, it may also contain excitatory currents generated by active dendritic conductances, activated, for example, by the combination of thalamic and cortical inputs or synchronous activity. While our use of voltage clamp reduces such effects, we cannot rule out the possibility of such amplification.

Our finding that $\mathrm{Q}_{\mathrm{Sub}}$ is orientation selective is consistent with the observation, in L2/3 of mouse visual cortex, that neurons tuned to the same orientation are more likely to excite each other ${ }^{12}$. Our data suggest that such a rule may also apply in L4 although the relatively large $\mathrm{Q}_{\mathrm{Sub}}$ at non-preferred orientations in some cells implies that the cortical connectivity is not exclusively orientation-specific. The fact that EPSC $_{\text {Sub }}$ is F1 modulated and that this F1 modulation is highly orientation selective may further imply specific connectivity among 
cortical neurons that are not only tuned to the same orientation but also share similar temporal phase. Novel methods for mapping the synaptic connectivity between functionally characterized neurons will address this possibility in the future ${ }^{12,30-34}$.

In conclusion, the fact that $\mathrm{F} 1_{\text {Sub }}$ and $\mathrm{F} 1_{\text {Thal }}$ are tuned to the same orientation and share the same temporal phase indicates that in the cortex, the excitation provided by thalamic inputs is amplified. Future experiments that selectively silence recurrent excitatory cortical synapses will help elucidate the impact of cortical amplification on the output of cortical neurons.

\section{Methods}

All experimental procedures were conducted in accordance with the National Institutes of Health guidelines and with the approval of the Committee on Animal Care at the University of California, San Diego. Data collection and analysis were not performed blind to the conditions of the experiments.

\section{Animals}

We used male and female transgenic mice heterozygous for PV-Cre (Jackson Labs \#008069). Mice were bred by crossing homozygous PV-Cre C57/B6 fathers with ICR white wild-type mothers. All offspring had pigmented eyes. Mice were housed in a vivarium with a reversed light cycle at a maximum of 5 animals per cage. We report whole-cell voltage clamp recordings from 40 mice. Loose-patch recordings in Fig. $1 \mathrm{~b}$ are from 2 mice. Combined loose-patch and LFP recordings in Supplementary Fig. 2 are from 4 mice. LFP recordings during pharmacological block of $\mathrm{GABA}_{\mathrm{B}}$ receptors in Supplementary Fig. 3 are from 6 mice. Thalamic units in Supplermentary Fig. 4 are from 3 mice.

\section{Virus injection}

Adeno-associated virus for Cre-dependent ChR2 expression

(AAV2/1.CAGGS.flex.ChR2.tdTomato.SV40, Addgene 18917) was obtained from the University of Pennsylvania Viral Vector Core. Virus was injected into the right visual cortex of neonatal PV-Cre mice between postnatal day 0 and 2 as previously described ${ }^{17,20}$. Pups were anesthetized on a cold pad $\left(0^{\circ} \mathrm{C}\right)$. Virus was loaded into a beveled glass micropipette (tip diameter 20-40 $\mu \mathrm{m}$ ) mounted on a Nanoject II (Drummond) attached to a micromanipulator. Three bolus injections of $23 \mathrm{nl}$ were made at a depth of 300-500 $\mu \mathrm{m}$ at each of three sites along the medial-lateral axis of $\mathrm{V} 1$.

\section{Animal preparation for in vivo physiology}

In vivo physiology experiments were performed on mice 1-3 months after neonatal virus injection. Mice were anesthetized by intraperitoneal injection of $1.5 \mathrm{~g} / \mathrm{kg}$ urethane and 2-4 $\mathrm{mg} / \mathrm{kg}$ chlorprothixene. During surgery this was supplemented by $1 \%$ isoflurane in $\mathrm{O}_{2}$. Depth of anesthesia was monitored with toe-pinch response. $3 \mathrm{mg} / \mathrm{kg}$ dexamethosone was administered subcutaneously to reduce brain swelling. A thin layer of silicon oil was applied to the eyes to prevent drying. The scalp and fascia were removed and a metal headplate was mounted over the right hemisphere using dental cement mixed with black paint. A 2-3 mm 
diameter craniotomy was performed over V1 (centered $2.5 \mathrm{~mm}$ lateral to the midline, $1 \mathrm{~mm}$ anterior to the lambda suture). In whole-cell recording experiments, a partial durotomy was performed on the lateral side of the craniotomy using a hooked 27-gauge needle for insertion of patch pipettes. Otherwise the dura was left intact. A thin layer of $1.5 \%$ low melting point agarose dissolved in ACSF (in mM: $140 \mathrm{NaCl}, 5 \mathrm{KCl}, 10$ D-Glucose, $10 \mathrm{HEPES}, 2 \mathrm{CaCl}_{2}, 2$ $\mathrm{MgSO}_{4}, \mathrm{pH}$ 7.4) was applied to the brain surface to reduce movement. The craniotomy was then kept submerged under a well of ACSF. Isoflurane was adjusted to $0-0.5 \%$ upon completion of craniotomy and/or durotomy surgery. At the end of the recording session, which lasted 4-8 hours, mice were deeply anesthetized with 5\% isoflurane and euthanized by decapitation.

\section{In vivo physiology}

Loose-patch recordings were obtained using glass patch pipettes (3-5 M $\Omega$ tip resistance) filled with $50 \mu \mathrm{M}$ Alexa 488 hydrazide in ACSF. Recordings were targeted to ChR2tdTomato negative neurons in L4 (350-450 $\mu$ m below the pia surface) using the shadowpatch technique ${ }^{35}$ under a two photon laser scanning microscope (Sutter) coupled to a Ti:Sapphire laser (Coherent, Ultra II) tuned to $900-1,000 \mathrm{~nm}$. Seal resistance of the loose patch was 10-100 M $\Omega$. Spikes were recorded in current-clamp mode with zero holding current.

Whole-cell recordings were obtained using the blind patch technique ${ }^{36}$. Whole-cell patch pipettes (3-5 M $\Omega$ tip resistance) were filled with $\mathrm{K}^{+}$based internal solution (in mM: $135 \mathrm{~K}$ gluconate, $8 \mathrm{NaCl}, 10 \mathrm{HEPES}, 4 \mathrm{Mg}$-ATP, 0.3 Na-GTP, 0.3 EGTA, pH 7.4) and $50 \mu \mathrm{M}$ Alexa 488 hydrazide. The depth of the recorded neuron was obtained upon termination of the recording by identifying the soma and/or pipette tip under two photon microscopy and measuring the distance to the pial surface. Neurons were voltage clamped at the reversal potential of inhibition ( $-68 \pm 0.8-69 \pm 0.8 \mathrm{mV}, \mathrm{n}=49$ ) to record excitatory postsynaptic currents (EPSCs). The reversal potential of inhibition was determined by adjusting the holding potential to minimize the amplitude of the IPSC evoked by photostimulation of PV cells. Average series resistance across the duration of the recording was $49 \pm 3 \mathrm{M} \Omega(\mathrm{n}=49)$. In 41/49 whole-cell recordings, local field potential (LFP) was concurrently monitored with a patch pipette (3-5 $\mathrm{M} \Omega$ tip resistance, $50 \mu \mathrm{M}$ Alexa 488 hydrazide in ACSF internal solution) in L2/3 (150-250 $\mu \mathrm{m}$ below the pia) in current clamp mode.

\section{Visual stimulation}

Stimuli were created using Matlab with the Psychophysics Toolbox ${ }^{37}$ were displayed on a gamma-corrected LCD monitor (Dell, $44 \times 27 \mathrm{~cm}, 60 \mathrm{~Hz}$ refresh rate, mean luminance 85 $\mathrm{cd} / \mathrm{m}^{2}$ ). The monitor was positioned $20 \mathrm{~cm}$ from the contralateral eye and repositioned such that the receptive field of the recorded neuron (as assessed by an experimenter-controlled white bar) was approximately centered. The ipsilateral eye was obscured from the monitor with a shield made of black tape.

Drifting gratings-Full field, full contrast drifting bar gratings with spatial frequency of 0.04 cycles per degree and temporal frequency of $2 \mathrm{~Hz}$ were displayed. Gratings were 
randomly presented at 12 evenly spaced directions. Stimulus duration was 1.5 or $1.7 \mathrm{~s}$ with a $2 \mathrm{~s}$ interstimulus interval in which a gray screen of mean luminance was displayed.

Receptive field mapping-The stimuli used to map receptive fields consisted of individually presented black (minimum luminance) or white squares (full luminance) against a gray background of mean luminance. The squares were 5 degrees in width and appeared in one of 64 locations of an $8 \times 8$ square grid covering $40 \times 40$ degrees. Stimuli were displayed for $100 \mathrm{~ms}$ followed by $200 \mathrm{~ms}$ of gray background before the next stimulus. Stimuli were presented in blocks of 5 or 19 with a $3.5 \mathrm{~s}$ or $1.5 \mathrm{~s}$ inter-block interval, respectively. A gray screen was presented during the inter-block interval. For each stimulus presentation, the location and luminance of the square was randomized.

\section{Photostimulation of PV cells}

ChR2-expressing PV cells were photostimulated using a $470 \mathrm{~nm}$ blue LED (Thorlabs) coupled to the widefield epifluorescence illumination pathway of the two photon microscope. The LED illumination was delivered to the brain via a $20 \times$ water-immersion objective (1.0 NA, Olympus). The field of view of the objective was centered on the recorded neuron and focused at its depth coordinate. The total power out of the front of the objective was 1.3 or $2.3 \mathrm{~mW}$. For drifting gratings and the 5-block flashed squares, the LED was turned on $0.64 \mathrm{~s}$ before the onset of visual stimulus and lasted $2.6 \mathrm{~s}$. For the 19-block receptive field stimuli, the LED was turned on $0.57 \mathrm{~s}$ before the onset of visual stimulus and lasted $6.4 \mathrm{~s}$. Thus the entire duration of visual stimulation was encompassed by the LED illumination period. Trials or blocks alternated between visual stimulus and LED + visual stimulus conditions.

To estimate the change in input resistance in the recorded neurons during PV cell photostimulation, cells were clamped at $2-4$ different potentials (range: -50 to $-80 \mathrm{mV}$ ) during an LED step lasting $1.8 \mathrm{~s}$. The input resistance prior to LED onset was calculated by determining the current at each potential, fitting a line to the current-voltage relationship and determining the inverse of the slope of that line. The current prior to LED onset was quantified as the bottom $5^{\text {th }}$ percentile of the current amplitude distribution to exclude spontaneous synaptic activity. Input resistance during the LED step was determined by plotting the average current during the last $1.1 \mathrm{~s}$ of LED illumination against the holding potential, performing a linear fit and determining the inverse of the slope of that fit.

\section{Data analysis}

All analysis was performed using custom written code in IgorPro (Wavemetrics).

Drifting gratings-The holding current (baseline, Fig. 3a top right) in a $540 \mathrm{~ms}$ window prior to the onset of visual stimulus was subtracted for each trial. This holding current was computed from the bottom $5^{\text {th }}$ percentile of the distribution of current values, which should include the periods with the least amount of spontaneous excitatory activity. This was confirmed by visual inspection. Trials with the same visual stimulus and LED conditions were then averaged together (3-8 repeats per cell). Trial-averaged synaptic currents recorded during control conditions without LED illumination were designated $\mathrm{EPSC}_{\mathrm{Tot}}$ and 
those recorded during LED illumination were designated $\mathrm{EPSC}_{\text {Thal }}$. $\mathrm{EPSC}_{\mathrm{Sub}}$ was generated by point-by-point subtraction of $\mathrm{EPSC}_{\text {Thal }}$ from $\mathrm{EPSC}_{\text {Tot }}\left(\mathrm{EPSC}_{\mathrm{Sub}}=\mathrm{EPSC}_{\mathrm{Tot}}-\mathrm{EPSC}_{\text {Thal }}\right)$.

The excitatory charge $(\mathrm{Q})$ was quantified as the time integral of the EPSC during the visual stimulus period (Fig. 3a, top right). F1 modulation was quantified by fitting a sinusoidal function with a periodicity matching the temporal frequency of the drifting grating $(2 \mathrm{~Hz})$ to the cycle-averaged response (average of 2-3 grating cycles; grating period $0.5 \mathrm{~s}$ ). The results of this fit were used to derive F1 amplitude and phase values (Fig. 3a, bottom right).

Orientation selectivity index (OSI) was calculated as 1 - circular variance ${ }^{38}$ using the following equation:

$$
O S I=\frac{\sqrt{\left(\sum r_{k} \sin \left(2 \theta_{k}\right)\right)^{2}+\left(\sum r_{k} \cos \left(2 \theta_{k}\right)\right)^{2}}}{\sum r_{k}}
$$

Where $r_{k}$ is the response to the $k$ th direction given by $\theta_{k}$. Direction selectivity index (DSI) was calculated as $\left(R_{\max }-R_{\operatorname{null}}\right) /\left(R_{\max }\right)$ where $\mathrm{R}_{\max }$ is the response to the stimulus that produced the maximum response and $R_{\text {null }}$ is the response to the direction 180 degrees away from that stimulus. As additional measures of orientation tuning, we also calculated the depth of modulation by orientation as well as the tuning width (Supplementary Fig. 5). The results for these measures of tuning are similar to those obtained by the OSI.

The population tuning curve for a particular parameter (i.e., Q or F1) was constructed by circularly shifting the tuning curves of all cells so that the preferred direction of a reference parameter occurred at 0 degrees and then averaging the tuning curves of all cells. The preferred direction was defined as the stimulus direction that evoked the largest response. The reference parameter could be the same or different from the parameter being plotted in the tuning curve. In figures showing population tuning curves of multiple parameters plotted on the same axis, if the population tuning curves were aligned to their own preferred direction, they are referred to as "self reference". Otherwise, the tuning curves were aligned to the preferred direction of a common reference parameter stated in the figure.

Preferred orientation was calculated as the vector average of responses ${ }^{39}$ using the following equation:

$$
\begin{gathered}
a=\sum r_{k} \cos \left(2 \theta_{k}\right) ; b=\sum r_{k} \sin \left(2 \theta_{k}\right) \\
\text { Preferred orientation }=0.5 \arctan (b / a) \text { if } a>0 \\
\text { Preferred orientation }=180+0.5 \arctan (b / a) \text { if } a<0
\end{gathered}
$$

Where $r_{k}$ is the response to the $k$ th direction given by $\theta_{k}$. Preferred orientation spanned the range of 0 to 180 degrees. Differences in preferred orientation were calculated by taking the absolute value of the difference. If this value was greater than 90 degrees, then it was adjusted to the complementary angle by subtracting it from 180 . Preferred direction was defined as the stimulus that produced the maximum response and spanned 0 to 330 degrees. Difference in preferred direction was calculated by taking the absolute value of the 
difference. If this value was greater than 180 degrees, then it was adjusted to the complementary angle by subtracting it from 360 .

Response to flashed squares-Responses to the same stimulus luminance, location, and LED conditions were averaged together (3-10 repeats per cell). The baseline activity for each average, defined as the average current level in a $30 \mathrm{~ms}$ window following stimulus onset was subtracted from each trace. We chose a late baseline window to limit the influence of large spontaneous fluctuations that often occurred during control trials in the absence of cortical silencing. While the baseline window occurred after the onset of the stimulus, it did not contain visually-evoked activity, as the latency to response was typically around $40 \mathrm{~ms}$.

Receptive field of thalamic excitation-Raw receptive field maps of thalamic excitation were obtained from the average response to black (OFF) or white (ON) squares at each grid location during cortical silencing. The response was taken as the integrated excitatory charge across a response window starting $40 \mathrm{~ms}$ after stimulus onset and lasting $100 \mathrm{~ms}$. This window allowed us to capture the response to the onset of the stimulus and avoid potential contamination by offset responses, which were occasionally observed. The response at each grid location was converted to a gray value to generate $8 \times 8$ heat maps of the raw receptive field. For each $\mathrm{ON}$ or OFF receptive field map, a set of background pixels was selected by eye that was clearly outside of the responsive region. The average value and standard deviation of the background pixels were calculated. Then, a $3 \times 3$ bilinear interpolation of the raw receptive field map was generated and z-scored using the previously calculated average and standard deviation of the background pixels. All pixels less than +2.5 z-scores were set to 0 . Contiguous non-zero regions with area $>2$ grid locations were designated as subfields. All other regions were set to 0 . The interpolated and thresholded maps of the subfields were used for subsequent analysis and display in contour plots.

Subfield area was calculated by counting all of the pixels in the subfield. Overlap area was the area of the region of overlap between $\mathrm{ON}$ and OFF subfields. Overlap index was equal to the overlap area as a fraction of the smaller subfield. The peak location of a subfield was determined as the center of mass of the set of pixels within 2-zscores of the strongest pixel. The subfield width along a given axis was defined as the linearly-interpolated full-width at half maximum of the subfield along that axis (as shown in the profile plots in Fig. 2c-d). This was calculated for both the axis between the ON and OFF subfields and the orthogonal axis. $\mathrm{RF}_{\text {Pref }}$, the preferred orientation predicted from the receptive field structure, was calculated as the orientation corresponding to gratings whose stripes are elongated perpendicular to the axis connecting the peak locations of the ON and OFF subfields. As shown in Figure 4d, horizontal gratings correspond to 0 degrees with clockwise rotations being positive.

Four neurons exhibited an OFF thalamic subfield but lacked sufficiently large ON responses to define an ON subfield according to the aforementioned criteria. In one case, the OFF thalamic subfield was situated on the edge of the grid used for receptive field mapping and so the presence or absence of an $\mathrm{ON}$ subfield could not be determined.

Simulation of separation between identical receptive field subfields-To ensure that the separation between the peaks of ON and OFF subfields that we measured was not 
due to noise in the estimate of peak location, we simulated the probability that a pair of identical subfields would exhibit a similar degree of peak separation given the variability of responses measured in pixels outside of the receptive field. For each subfield ( $n=26$ from 13 cells with $1 \mathrm{ON}$ and 1 OFF subfield), the raw 8 pixel $\times 8$ pixel heat map served as the underlying model subfield for our simulations. A noise value was added to each pixel to generate one simulated subfield. The noise was drawn from a Gaussian distribution centered at 0 with a standard deviation equal to the standard deviation of the responses in the background pixels (as defined in the previous section). The location of the peak of the simulated subfield was determined as stated in the previous section. The distance between peak locations for 1,000 pairs of simulated subfields (each starting from the same "true" subfield) was determined. The probability that the measured ON-OFF separation for a cell was less than the separation between simulated identical subfields was calculated as the fraction of simulated distances that was greater than or equal to the measured values. Because each cell had an ON and an OFF subfield, we generated 2 probability values for each cell that used either the ON or the OFF subfield as the model receptive field. We refer to the larger of the two probability values in the text.

\section{Statistical analysis}

All error bars are presented as mean \pm s.e.m and statistical significance was determined using two-sided paired t-tests unless otherwise stated. No statistical methods were used to predetermine sample sizes, but our sample sizes are similar to those reported in previous publications in the field ${ }^{3,16,21}$. Data distribution was assumed to be normal but this was not formally tested.

\section{Supplementary Material}

Refer to Web version on PubMed Central for supplementary material.

\section{Acknowledgments}

We thank J. Evora for help with genotyping and mouse husbandry, J. Isaacson, E. Chichilnisky, and the members of the Scanziani and Isaacson labs for helpful discussions of this project, and S. Olsen and K. Reinhold for help with dLGN recordings. This project was supported by the Gatsby charitable foundation, the Brain \& Behavior Research Foundation and the Howard Hughes Medical Institute.

\section{References}

1. Nelson S, Toth L, Sheth B, Sur M. Orientation selectivity of cortical neurons during intracellular blockade of inhibition. Science. 1994; 265:774-7. [PubMed: 8047882]

2. Anderson JS, Carandini M, Ferster D. Orientation tuning of input conductance, excitation, and inhibition in cat primary visual cortex. Journal of neurophysiology. 2000; 84:909-26. [PubMed: 10938316]

3. Liu BH, et al. Intervening inhibition underlies simple-cell receptive field structure in visual cortex. Nature neuroscience. 2010; 13:89-96. [PubMed: 19946318]

4. Liu BH, et al. Broad inhibition sharpens orientation selectivity by expanding input dynamic range in mouse simple cells. Neuron. 2011; 71:542-54. [PubMed: 21835349]

5. Brecht M, Sakmann B. Dynamic representation of whisker deflection by synaptic potentials in spiny stellate and pyramidal cells in the barrels and septa of layer 4 rat somatosensory cortex. The Journal of physiology. 2002; 543:49-70. [PubMed: 12181281] 
6. Liu BH, Wu GK, Arbuckle R, Tao HW, Zhang LI. Defining cortical frequency tuning with recurrent excitatory circuitry. Nature neuroscience. 2007; 10:1594-600. [PubMed: 17994013]

7. Hubel DH, Wiesel TN. Receptive fields, binocular interaction and functional architecture in the cat's visual cortex. The Journal of physiology. 1962; 160:106-54. [PubMed: 14449617]

8. Chapman B, Zahs KR, Stryker MP. Relation of cortical cell orientation selectivity to alignment of receptive fields of the geniculocortical afferents that arborize within a single orientation column in ferret visual cortex. The Journal of neuroscience. 1991; 11:1347-58. [PubMed: 2027051]

9. Jin J, Wang Y, Swadlow HA, Alonso JM. Population receptive fields of ON and OFF thalamic inputs to an orientation column in visual cortex. Nature neuroscience. 2011; 14:232-8. [PubMed: 21217765]

10. Reid RC, Alonso JM. Specificity of monosynaptic connections from thalamus to visual cortex. Nature. 1995; 378:281-4. [PubMed: 7477347]

11. Usrey WM, Alonso JM, Reid RC. Synaptic interactions between thalamic inputs to simple cells in cat visual cortex. The Journal of neuroscience. 2000; 20:5461-7. [PubMed: 10884329]

12. Ko H, et al. Functional specificity of local synaptic connections in neocortical networks. Nature. 2011; 473:87-91. [PubMed: 21478872]

13. Gilbert CD, Wiesel TN. Columnar specificity of intrinsic horizontal and corticocortical connections in cat visual cortex. The Journal of neuroscience. 1989; 9:2432-42. [PubMed: 2746337]

14. Malach R, Amir Y, Harel M, Grinvald A. Relationship between intrinsic connections and functional architecture revealed by optical imaging and in vivo targeted biocytin injections in primate striate cortex. Proceedings of the National Academy of Sciences of the United States of America. 1993; 90:10469-73. [PubMed: 8248133]

15. Bosking WH, Zhang Y, Schofield B, Fitzpatrick D. Orientation selectivity and the arrangement of horizontal connections in tree shrew striate cortex. The Journal of neuroscience. 1997; 17:211227. [PubMed: 9045738]

16. Ferster D, Chung S, Wheat H. Orientation selectivity of thalamic input to simple cells of cat visual cortex. Nature. 1996; 380:249-52. [PubMed: 8637573]

17. Olsen SR, Bortone DS, Adesnik H, Scanziani M. Gain control by layer six in cortical circuits of vision. Nature. 2012; 483:47-52. [PubMed: 22367547]

18. Nagel G, et al. Channelrhodopsin-2, a directly light-gated cation-selective membrane channel. Proceedings of the National Academy of Sciences of the United States of America. 2003; 100:13940-5. [PubMed: 14615590]

19. Boyden ES, Zhang F, Bamberg E, Nagel G, Deisseroth K. Millisecond-timescale, genetically targeted optical control of neural activity. Nature neuroscience. 2005; 8:1263-8. [PubMed: 16116447]

20. Atallah BV, Bruns W, Carandini M, Scanziani M. Parvalbumin-expressing interneurons linearly transform cortical responses to visual stimuli. Neuron. 2012; 73:159-70. [PubMed: 22243754]

21. Chung S, Ferster D. Strength and orientation tuning of the thalamic input to simple cells revealed by electrically evoked cortical suppression. Neuron. 1998; 20:1177-89. [PubMed: 9655505]

22. Piscopo DM, El-Danaf RN, Huberman AD, Niell CM. Diverse visual features encoded in mouse lateral geniculate nucleus. The Journal of neuroscience. 2013; 33:4642-56. [PubMed: 23486939]

23. Grubb MS, Thompson ID. Quantitative characterization of visual response properties in the mouse dorsal lateral geniculate nucleus. Journal of neurophysiology. 2003; 90:3594-607. [PubMed: 12944530]

24. Marshel JH, Kaye AP, Nauhaus I, Callaway EM. Anterior-posterior direction opponency in the superficial mouse lateral geniculate nucleus. Neuron. 2012; 76:713-20. [PubMed: 23177957]

25. Lampl I, Anderson JS, Gillespie DC, Ferster D. Prediction of orientation selectivity from receptive field architecture in simple cells of cat visual cortex. Neuron. 2001; 30:263-74. [PubMed: 11343660]

26. Cudeiro J, Sillito AM. Looking back: corticothalamic feedback and early visual processing. Trends in neurosciences. 2006; 29:298-306. [PubMed: 16712965]

27. Niell CM, Stryker MP. Modulation of visual responses by behavioral state in mouse visual cortex. Neuron. 2010; 65:472-9. [PubMed: 20188652] 
28. Adesnik H, Bruns W, Taniguchi H, Huang ZJ, Scanziani M. A neural circuit for spatial summation in visual cortex. Nature. 2012; 490:226-31. [PubMed: 23060193]

29. Haider B, Häusser M, Carandini M. Inhibition dominates sensory responses in the awake cortex. Nature. 2013; 493:97-100. [PubMed: 23172139]

30. Lien AD, Scanziani M. In vivo Labeling of Constellations of Functionally Identified Neurons for Targeted in vitro Recordings. Frontiers in neural circuits. 2011; 5:16. [PubMed: 22144948]

31. Briggman KL, Helmstaedter M, Denk W. Wiring specificity in the direction-selectivity circuit of the retina. Nature. 2011; 471:183-8. [PubMed: 21390125]

32. Bock DD, et al. Network anatomy and in vivo physiology of visual cortical neurons. Nature. 2011; 471:177-82. [PubMed: 21390124]

33. Jia H, Rochefort NL, Chen X, Konnerth A. Dendritic organization of sensory input to cortical neurons in vivo. Nature. 2010; 464:1307-12. [PubMed: 20428163]

34. Marshel JH, Mori T, Nielsen KJ, Callaway EM. Targeting single neuronal networks for gene expression and cell labeling in vivo. Neuron. 2010; 67:562-74. [PubMed: 20797534]

35. Kitamura K, Judkewitz B, Kano M, Denk W, Häusser M. Targeted patch-clamp recordings and single-cell electroporation of unlabeled neurons in vivo. Nature methods. 2008; 5:61-7. [PubMed: 18157136]

36. Margrie TW, Brecht M, Sakmann B. In vivo, low-resistance, whole-cell recordings from neurons in the anaesthetized and awake mammalian brain. Pflügers Archive: European journal of physiology. 2002; 444:491-8. [PubMed: 12136268]

37. Brainard DH. The Psychophysics Toolbox. Spatial vision. 1997; 10:433-6. [PubMed: 9176952]

38. Ringach DL, Shapley RM, Hawken MJ. Orientation selectivity in macaque V1: diversity and laminar dependence. The Journal of neuroscience. 2002; 22:5639-51. [PubMed: 12097515]

39. Swindale N. V Orientation tuning curves: empirical description and estimation of parameters. Biological cybernetics. 1998; 78:45-56. [PubMed: 9518026] 
a

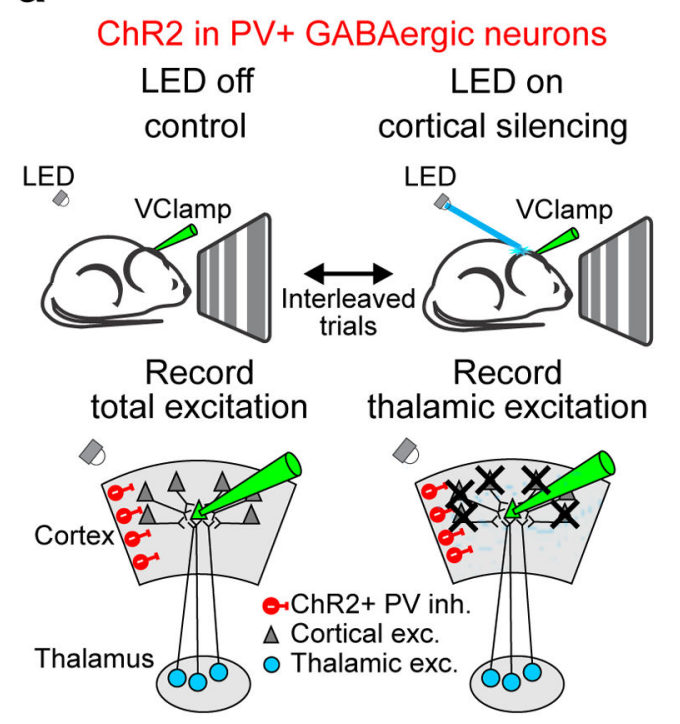

C

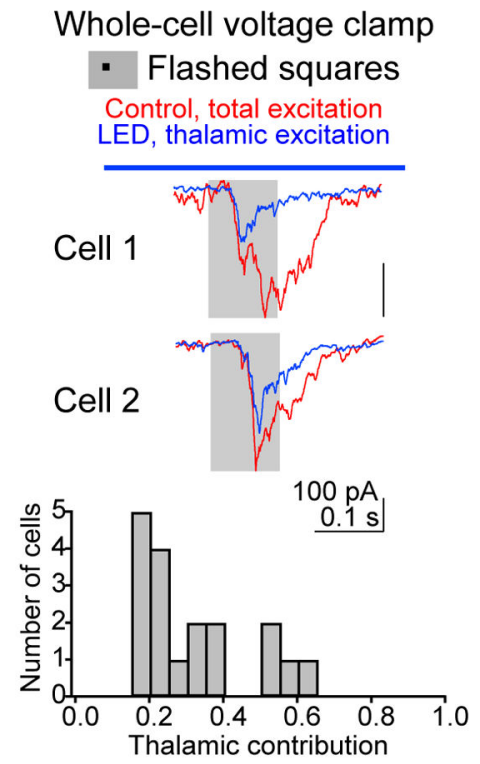

b

\section{Loose-patch recording \\ -lll Drifting gratings}

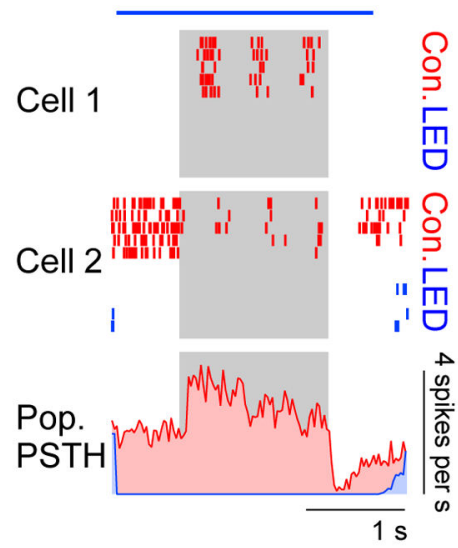

Whole-cell voltage clamp All Drifting gratings Control, total excitation LED, thalamic excitation

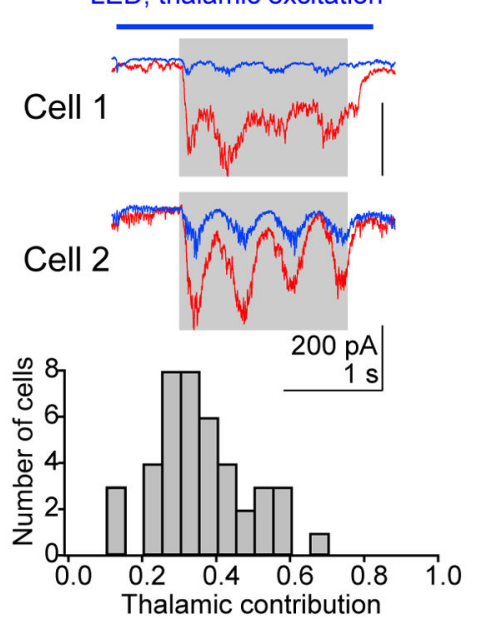

Figure 1. Isolating thalamic excitation

a, Schematic of experimental approach for isolating thalamic excitation recorded in response to visual stimulation in layer 4 neurons voltage clamped at the reversal potential for inhibition. Thalamic excitation is isolated by silencing cortical activity via photostimulation of ChR2-expressing PV cells. b, Optogenetic silencing of visually evoked cortical activity. Top: The spiking responses of two example cortical neurons recorded in the loose patch configuration with (LED, blue) and without (control, red) PV cell photostimulation are shown as raster plots. Gray rectangle, visual stimulus (1.5 s). Blue bar, LED illumination (2.6 s). Control and LED trials were interleaved, but separated here for clarity. Bottom, population peristimulus time histogram (PSTH) averaged across all cells and all stimulus directions ( $\mathrm{n}=14$ cells, 2 mice). Note complete suppression of spiking during LED 
illumination. The high spontaneous activity and its stimulus-evoked reduction in cell 2 were rare. c, Total and thalamic excitation in response to flashed squares. Top, visually evoked EPSCs recorded in two example cortical neurons with (blue) and without (red) cortical silencing in response to a flashed square (5 degrees) at optimal location and luminance. Average of 8 (cell 1) and 4 (cell 2) trials. Gray rectangle, visual stimulus. Blue bar, LED illumination. Bottom, distribution of the fraction of excitatory charge contributed by the thalamus ( $\mathrm{n}=18$ cells, 16 mice). $\mathbf{d}$, Same as $\mathbf{c}$ for drifting gratings. The response to the preferred direction is shown. Average of 8 (cell 1) and 5 (cell 2) trials. Bottom, distribution of the fraction of excitatory charge contributed by the thalamus ( $n=42$ cells, 33 mice). 
a Whole-cell voltage clamp during cortical silencing
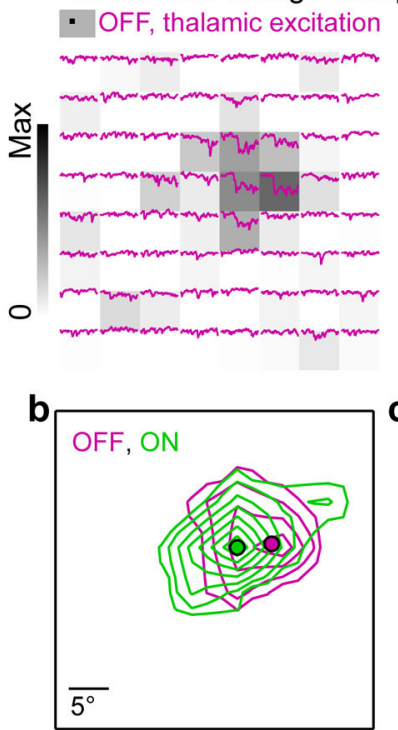

e
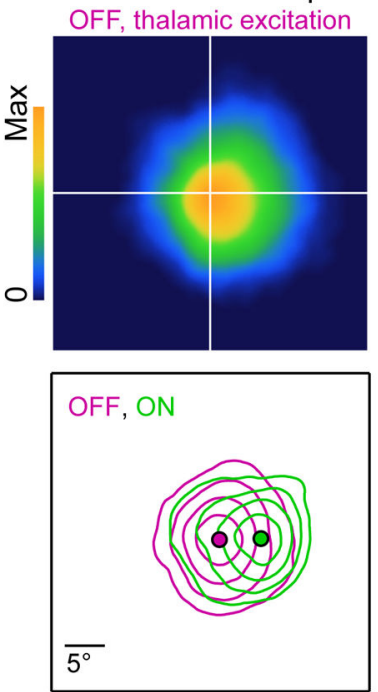

- ON, thalamic excitation
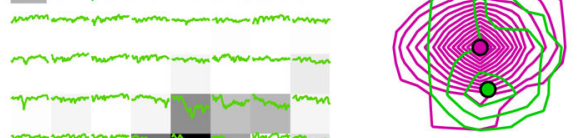

Ind
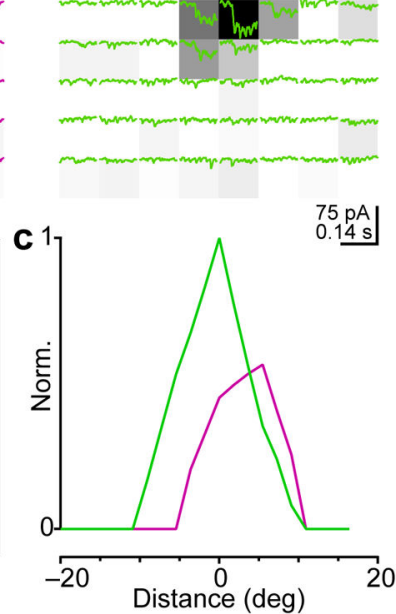

Population average

$\mathrm{ON}$, thalamic excitation

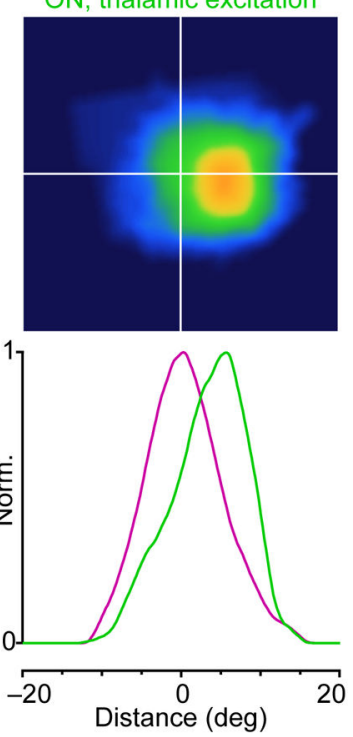

d
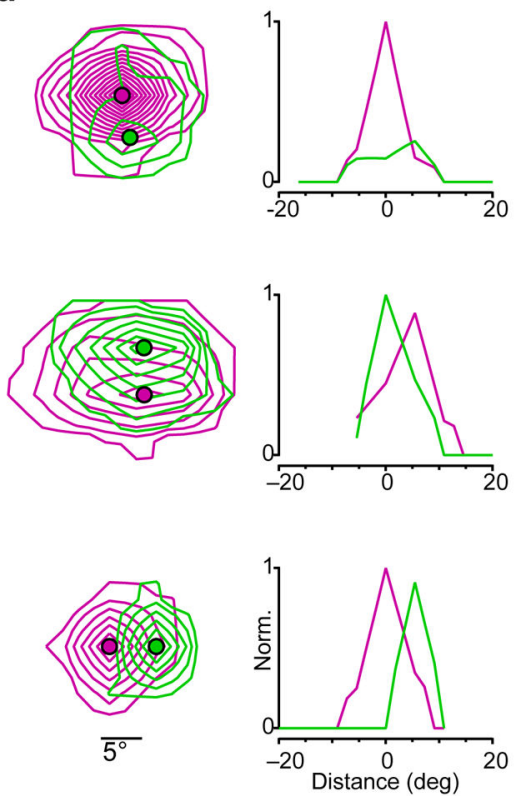

f

Subfield area

000

Subfield width $\leftrightarrow$ ON-OFF axis \begin{tabular}{lll}
\hline & 300 & 600 \\
$\mathrm{Deg}^{2}$ &
\end{tabular} 101
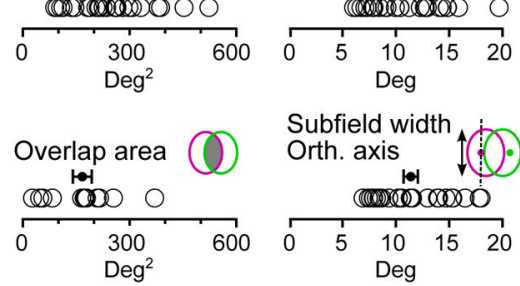

Subfield width Orth. axis (1)

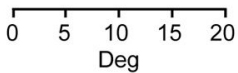

Overlap index
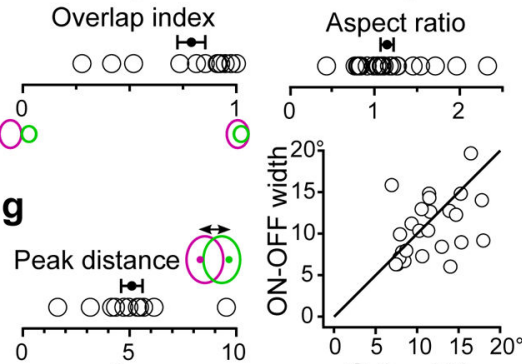

Figure 2. Receptive field structure of thalamic excitation

a, Example cell. Isolated thalamic excitation (EPSC $\left.{ }_{\text {Thal }}\right)$ in response to black ("OFF", magenta traces, left) or white ("ON", green traces, right) squares at each of 64 locations on an $8 \times 8$ grid. Stimuli appear at the beginning of each trace and last for $100 \mathrm{~ms}$. The raw receptive field heat maps calculated from the thalamic excitatory charge $\left(\mathrm{Q}_{\text {Thal }}\right)$ evoked at each grid location and normalized to the peak ON response are depicted by the background gray-level of each trace. Average of 4 trials per location. b, Contour plot of the OFF and ON subfields for the cell in $\mathbf{a}$. Each contour represents 2 z-scores. Filled magenta and green circles mark the peaks of the OFF and ON receptive fields, respectively. c, Profile plot of $\mathrm{OFF}$ and $\mathrm{ON}$ receptive fields in $\mathbf{c}$ along the axis connecting their peaks. $\mathbf{d}$, Same as b and $\mathbf{c}$ 
for 3 additional neurons. e, Population average of OFF and ON receptive fields. Before averaging, the receptive fields for each cell were centered on the peak of the OFF subfield and rotated so that the peak of the ON subfield was directly to the right of the OFF. OFF and ON subfields were separately normalized. Top, heat maps of the population average OFF and $\mathrm{ON}$ receptive fields. Bottom, contour and profile plots of the population average receptive fields as in $\mathbf{b}$ and $\mathbf{c}$. The outermost contour represents $10 \%$ of the peak and each additional contour is an increment of $20 \%$ of the peak. $\mathbf{f}$, Quantification of subfield area ( $\mathrm{n}=$ 26 subfields) and overlap between ON and OFF subfields ( $\mathrm{n}=13$ cells). $\mathrm{g}$, Distance between the peaks of ON and OFF subfields ( $n=13$ cells). $\mathbf{h}$, Comparison of subfield width along the axis connecting the ON and OFF peaks (ON/OFF axis) or the orthogonal axis ( $n=26$ subfields). Black line, unity. Data in $\mathbf{e}-\mathbf{h}$ are from 13 cells each exhibiting 1 OFF and 1 ON subfield from 12 mice. Error bars, mean \pm s.e.m. 
a Whole-cell voltage clamp during cortical silencing

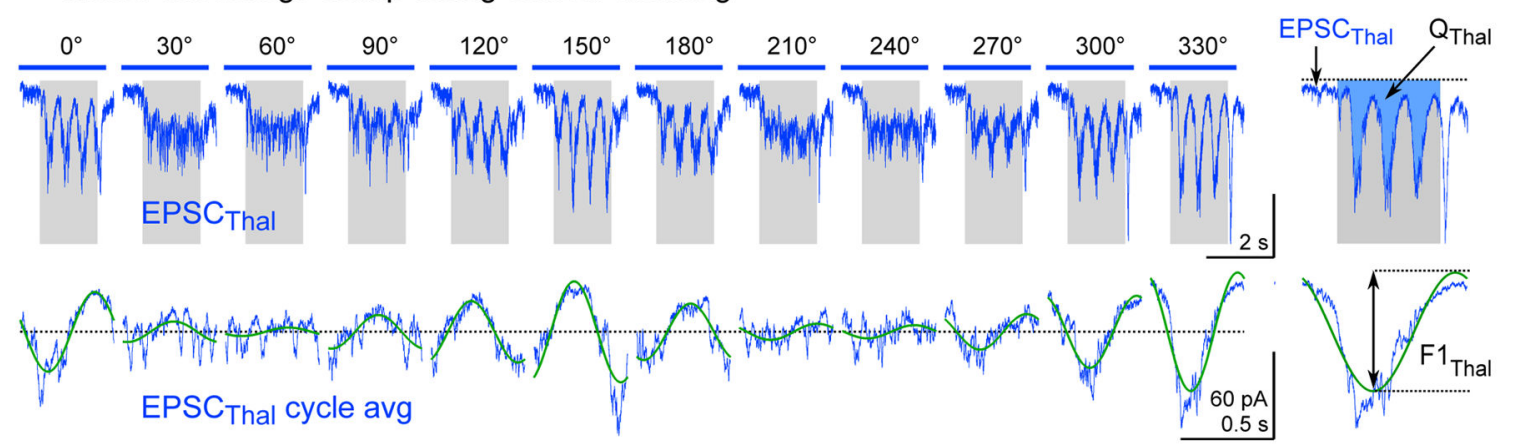

b Example cell

C

Population average

d Orientation e

Direction
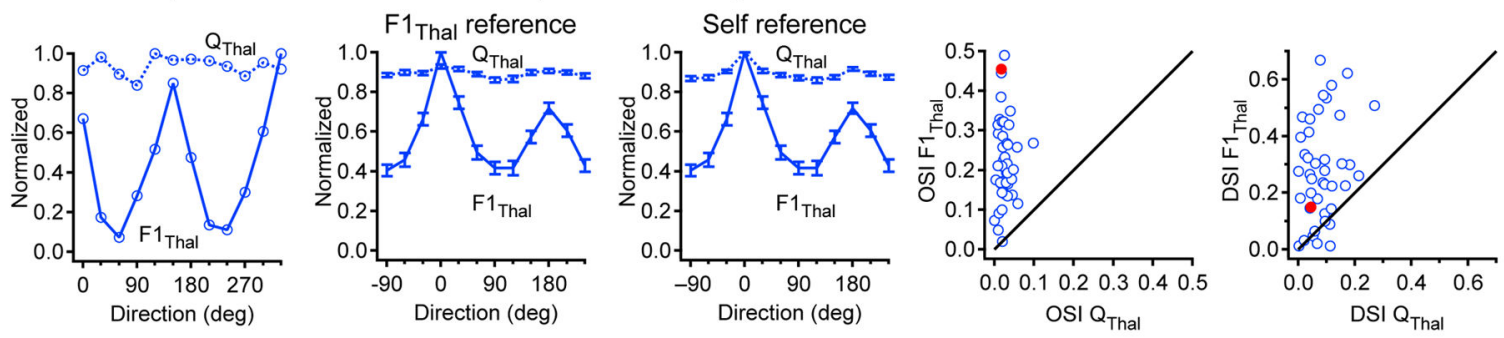

Figure 3. Orientation tuning of thalamic excitation

a, Example cell: Top, Isolated thalamic excitation (EPSC $\mathrm{Ehal}_{\text {Tha }}$ ) in response to drifting gratings of various orientations (average of 8 trials per direction). Gray rectangle, visual stimulus (1.7 s). Blue bar, LED illumination ( $2.6 \mathrm{~s})$. Bottom, F1 modulation of $\mathrm{EPSC}_{\text {Thal }}$. Cycle average (blue) and best-fitting sinusoid (green) at the grating temporal frequency (2 $\mathrm{Hz}$ ). The y-offset was removed to aid comparison of $\mathrm{F} 1$ amplitude across different orientations. Right, $\mathrm{EPSC}_{\text {Thal }}$ and cycle average in response to 330 degree grating at expanded time scale showing how $\mathrm{Q}_{\mathrm{Thal}}$ and $\mathrm{F} 1_{\text {Thal }}$ are determined. b, Orientation tuning curves of $\mathrm{Q}_{\text {Thal }}$ (dashed line) and $\mathrm{F} 1_{\text {Thal }}$ (solid line) for the neuron in (a). c, Population tuning curves of $\mathrm{Q}_{\text {Thal }}$ (dashed line) and $\mathrm{F}_{\text {Thal }}$ (solid line). Left, Population tuning curves in which $\mathrm{Q}_{\text {Thal }}$ and $\mathrm{F} 1_{\text {Thal }}$ tuning curves for each cell were equally shifted so that the preferred direction of $\mathrm{F}_{1}$ Thal occurred at 0 degrees $\left(\mathrm{F} 1_{\text {Thal }}\right.$ reference). Right, Population tuning curves in which $\mathrm{Q}_{\mathrm{Thal}}$ and $\mathrm{F}_{\text {Thal }}$ tuning curves for each cell were independently shifted so that preferred direction of $\mathrm{Q}_{\mathrm{Thal}}$ and $\mathrm{F} 1_{\text {Thal }}$ both occurred at 0 degrees (self reference). d, Orientation selectivity index (OSI) of $\mathrm{Q}_{\text {Thal }}$ plotted against OSI of F1 Thal for each cell. e, Direction selectivity index (DSI) of $\mathrm{Q}_{\text {Thal }}$ plotted against DSI of F1 $1_{\text {Thal }}$ for each cell. Filled red markers in $\mathbf{d}$ and $\mathbf{e}$ denote the OSI and DSI values of the example cell. Data in $\mathbf{c}-\mathbf{e}$ are from $n=42$ cells from 33 mice. Error bars, mean \pm s.e.m. 
a Whole-cell voltage clamp during cortical silencing
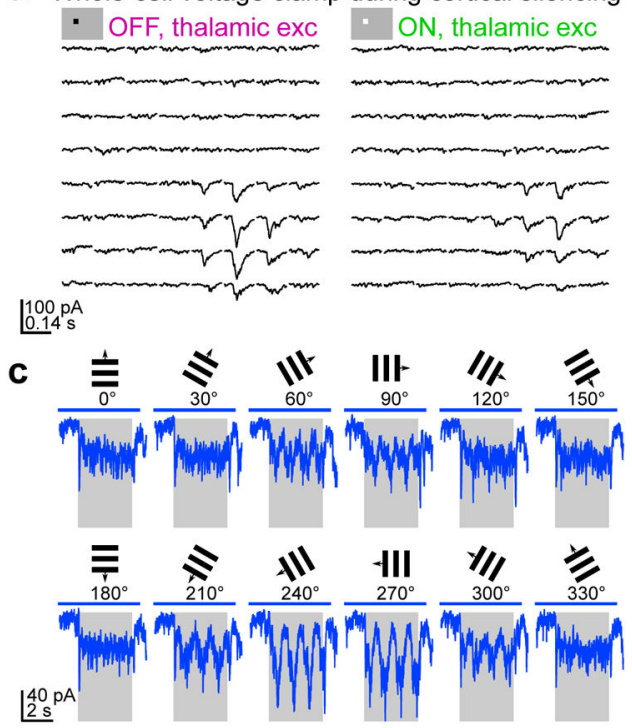

e
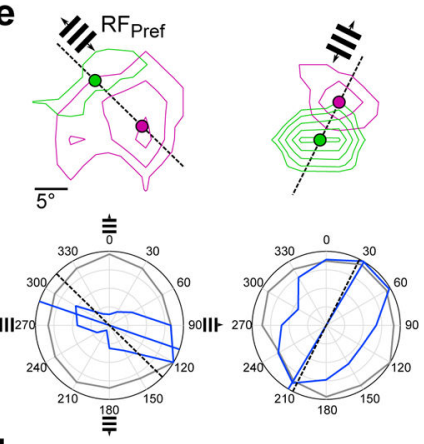

h

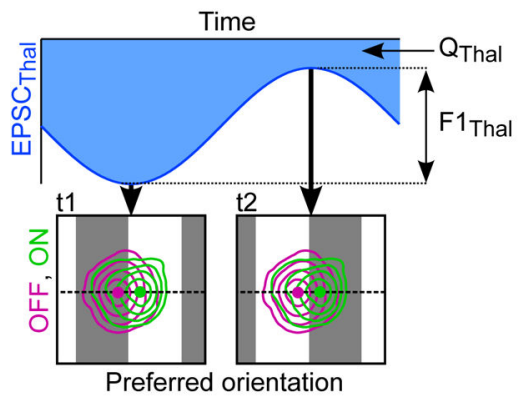

b

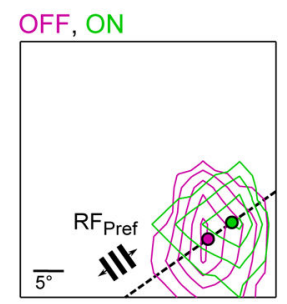

d
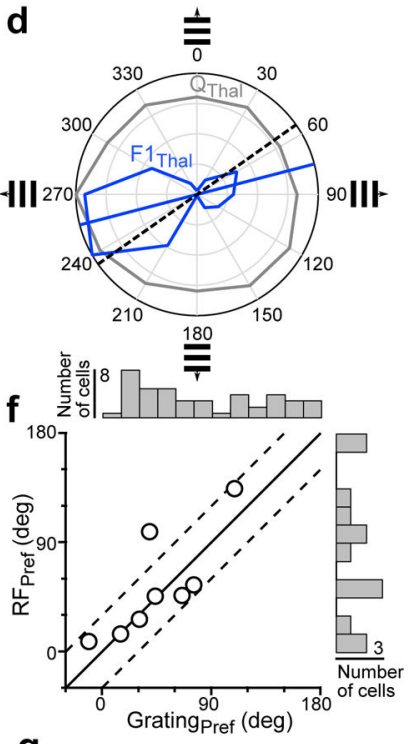

g
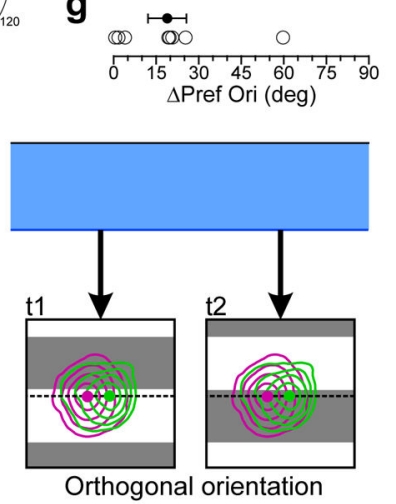

Figure 4. Separation of $\mathrm{ON}$ and $\mathrm{OFF}$ thalamic subfields predicts preferred orientation of thalamic excitation

a-d, Example recording of isolated thalamic excitation $\left(\mathrm{EPSC}_{\mathrm{Thal}}\right)$ where both the $\mathrm{ON}$ and OFF receptive fields and the responses to drifting gratings at various orientations were

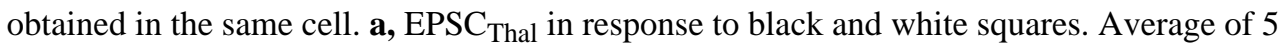
trials per location. $\mathbf{b}$, Contour plot of the OFF and $\mathrm{ON}$ receptive field maps for the cell in a. Each contour represents 2 z-scores. Filled magenta and green circles mark the peaks of the $\mathrm{OFF}$ and ON receptive fields, respectively. Dashed black line connects the OFF and ON peaks to define the ON-OFF axis. The preferred orientation predicted from the ON-OFF axis, $\mathrm{RF}_{\text {Pref }}$, is indicated by the small grating. $\mathbf{c}, \mathrm{EPSC}_{\mathrm{Thal}}$ in response to drifting gratings of various orientations (average of 3 trials per direction). Gray rectangle, visual stimulus (1.7 
s). Blue bar, LED illumination ( $2.6 \mathrm{~s}$ ). d, Orientation tuning curves of $\mathrm{F} 1_{\text {Thal }}$ (blue) and $\mathrm{Q}_{\text {Thal }}$ (gray) in polar coordinates for the responses in $\mathbf{c}$. The blue line indicates the preferred orientation of $F 1_{\text {Thal }}$ (Grating ${ }_{\text {Pref }}$ ) and the black dashed line corresponds to $\mathrm{RF}_{\text {Pref. }}$ e, Same as $\mathbf{b}$ and $\mathbf{d}$ for three additional cells. $\mathbf{f}, \mathrm{RF}_{\text {Pref }}$ plotted against Grating ${ }_{\text {Pref }}(\mathrm{n}=8$ cells, 7 mice). Black line, unity. The dashed lines denote the region in which cells the difference between $\mathrm{RF}_{\text {Pref }}$ and Grating $g_{\text {Pref }}$ is less than 30 degrees. The distributions of Grating ${ }_{\text {Pref }}(\mathrm{n}=$ 42, 33 mice) and $\operatorname{RF}_{\text {Pref }}(\mathrm{n}=13$ cells, 12 mice) across the population of cells in which either value was measured are shown along the top and right, respectively. g, Absolute difference in $\mathrm{RF}_{\text {Pref }}$ and Grating Pref $(\Delta$ Pref Ori) $(\mathrm{n}=8$ cells, 7 mice). Error bars, mean \pm s.e.m. $\mathbf{h}$, Diagram of how orientation tuning of $\mathrm{F}_{1}$ Thal arises from spatially offset $\mathrm{OFF}$ and $\mathrm{ON}$ thalamic excitatory input. The area of the blue shaded region corresponds to $\mathrm{Q}_{\mathrm{Thal}}$. The difference between the peak and the trough of $\mathrm{EPSC}_{\text {Thal }}$ corresponds to $\mathrm{F}_{\text {Thal }}$. 


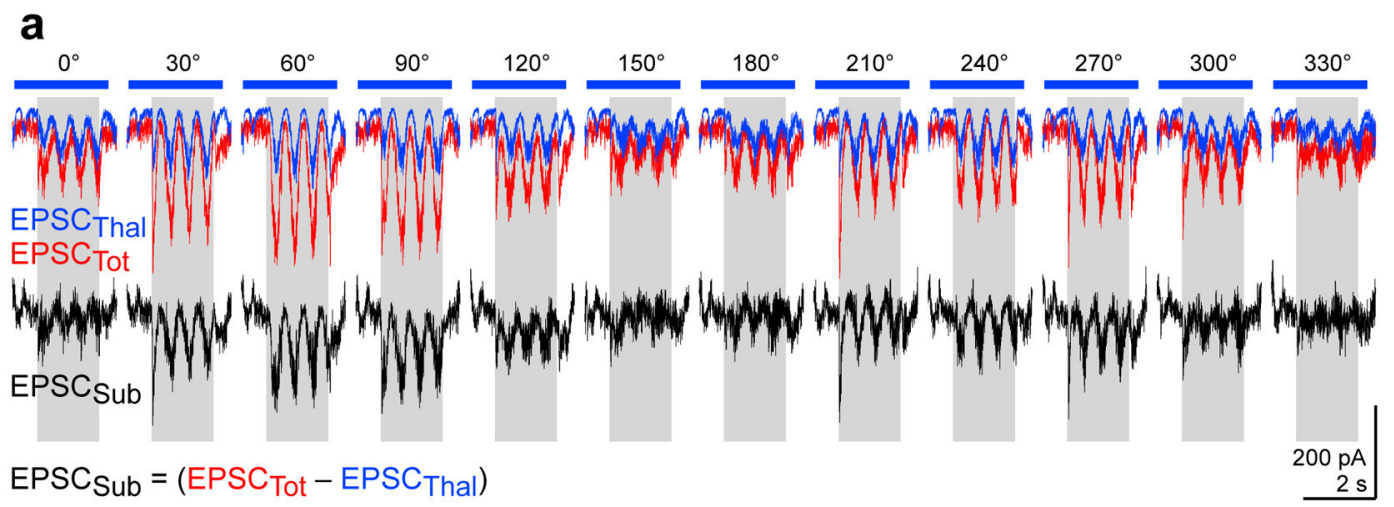

b Example cell

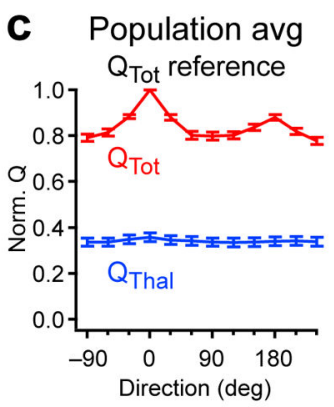

d Orientation

e Direction
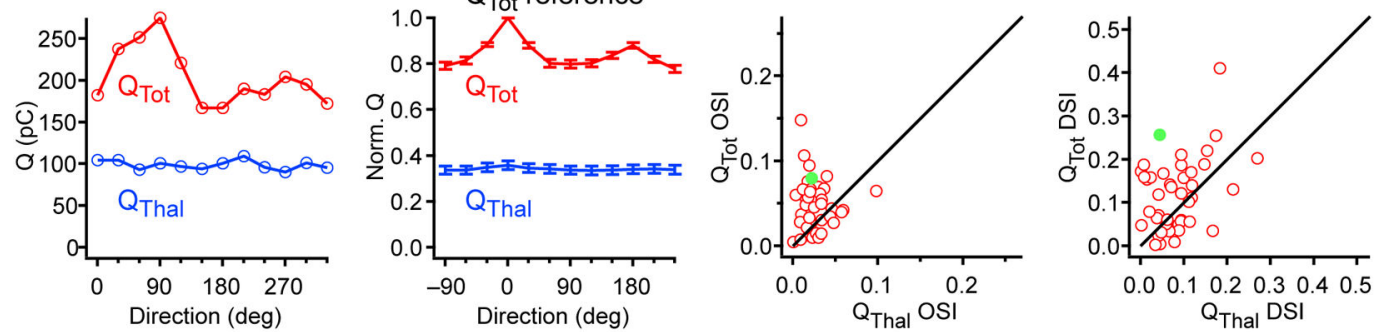

f Example cell

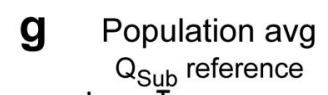

h Orientation
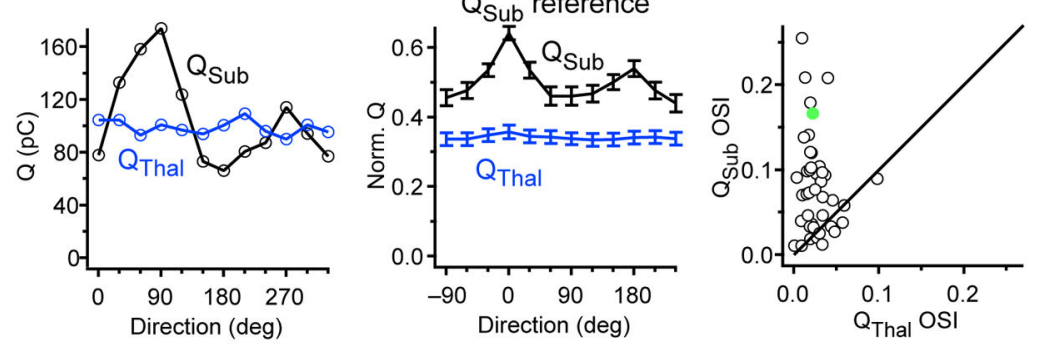

i

Direction

Figure 5. Tuning of non-thalamic excitatory charge

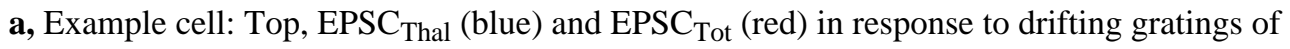
various orientations. Bottom, EPSC $_{\text {Sub }}$ derived from point-by-point subtraction of $\mathrm{EPSC}_{\mathrm{Thal}}$ from $\mathrm{EPSC}_{\mathrm{Tot}}$. Gray rectangle, visual stimulus (1.7 s). Blue bar, LED illumination (2.6 s). b, Orientation tuning curves of $\mathrm{Q}_{\mathrm{Tot}}(\mathrm{red})$ and $\mathrm{Q}_{\mathrm{Thal}}$ (blue) for the example cell in (a). c, Population tuning curves of $\mathrm{Q}_{\mathrm{T} \text { tot }}$ (red) and $\mathrm{Q}_{\mathrm{Thal}}$ (blue). Tuning curves are aligned to the preferred direction of $\mathrm{Q}_{\mathrm{Tot}}\left(\mathrm{Q}_{\text {tot }}\right.$ reference) and normalized by the value of $\mathrm{Q}_{\mathrm{T} \text { ot }}$ at its preferred direction. d, OSI of $\mathrm{Q}_{\text {Tot }}$ plotted against OSI of $\mathrm{Q}_{\mathrm{Thal}}$ for all neurons. e, DSI of $\mathrm{Q}_{\text {Tot }}$ plotted against DSI of $\mathrm{Q}_{\text {Thal }}$ for all neurons. f-i, Same as (b-e) for $\mathrm{Q}_{\text {Sub }}$ (black) and $\mathrm{Q}_{\text {Thal }}$ (blue). Filled green markers in $\mathbf{d}, \mathbf{e}, \mathbf{h}$, and $\mathbf{i}$ denote the OSI and DSI values of the example cell. Data in $\mathbf{c}-\mathbf{e}$ and $\mathbf{g - i}$ are from $\mathrm{n}=42$ cells from 33 mice. Error bars, mean \pm s.e.m. 


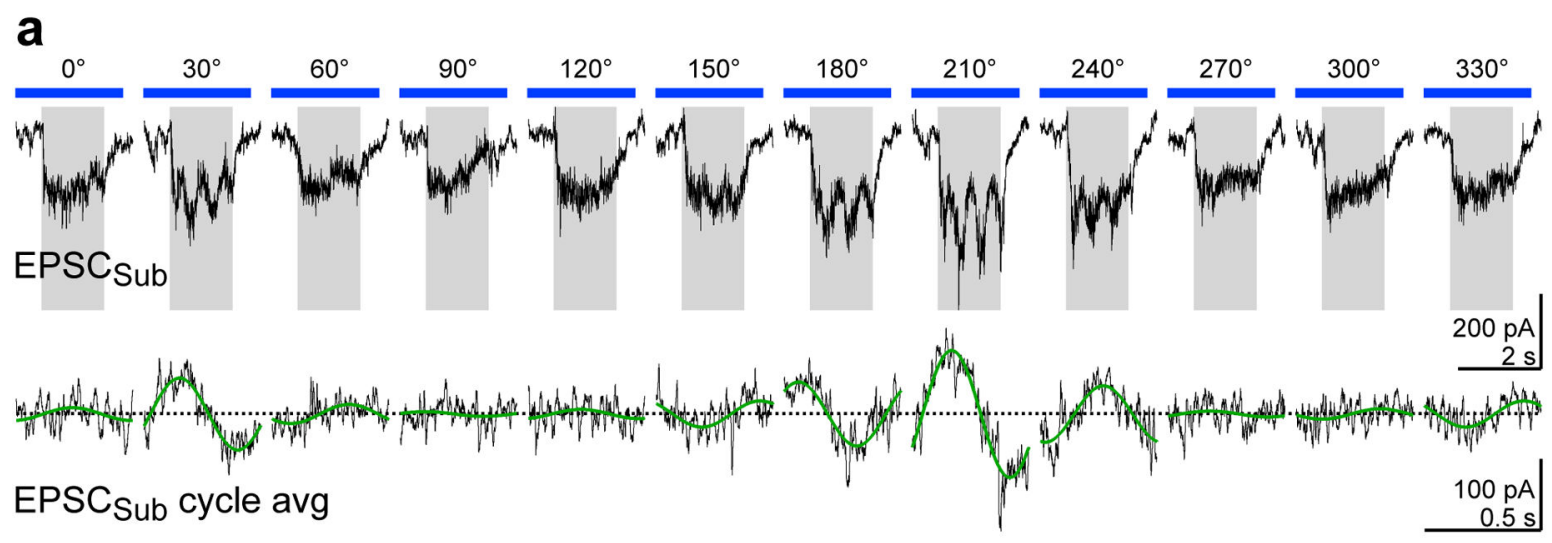

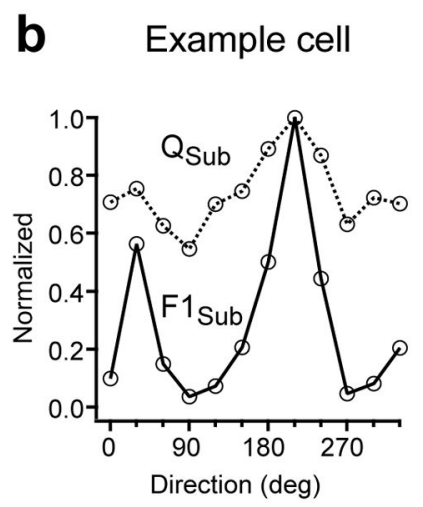
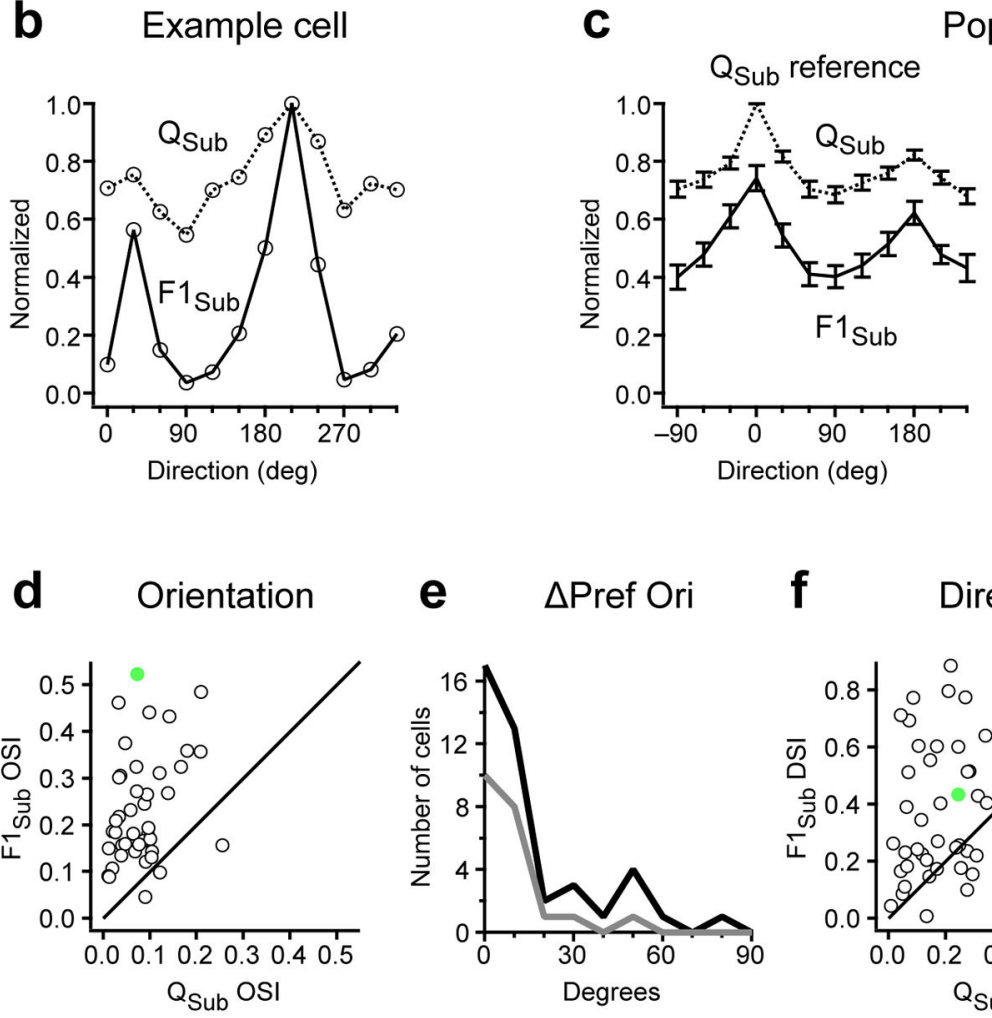
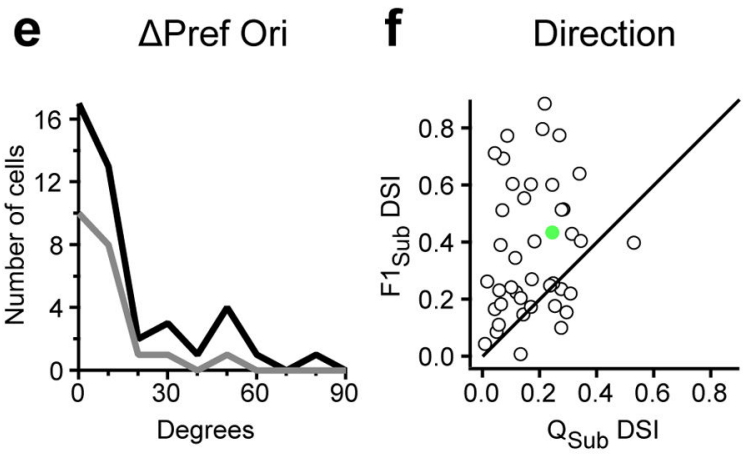

Figure 6. Tuning of non-thalamic excitatory F1 modulation

a, Example cell: Top, EPSC $_{\text {Sub }}$ in response to drifting gratings of various orientations. Gray rectangle, visual stimulus (1.5 s). Blue bar, LED illumination (2.6 s). Bottom, F1 modulation of EPSC $_{\text {Sub }}$. Cycle average (black) and best-fitting sinusoid (green) at the grating temporal frequency $\left(2 \mathrm{~Hz}\right.$ ). b, Orientation tuning curves of $\mathrm{Q}_{\mathrm{Sub}}$ (dashed curve) and $\mathrm{F} 1_{\text {Sub }}$ (solid curve) for the example cell in a. c, Population tuning curve of $\mathrm{Q}_{\text {Sub }}$ (dashed curve) and $\mathrm{F} 1_{\text {Sub }}$ (solid curve). Left, Population tuning curves in which $\mathrm{Q}_{\text {Sub }}$ and $\mathrm{F} 1_{\text {Sub }}$ tuning curves for each cell were equally shifted so that the preferred direction of $\mathrm{Q}_{\text {sub }}$ occurred at 0 degrees $\left(\mathrm{Q}_{\text {sub }}\right.$ reference). Right, Population tuning curves in which $\mathrm{Q}_{\text {Sub }}$ and $\mathrm{F} 1_{\text {Sub }}$ tuning curves for each cell were independently shifted so that preferred direction of $\mathrm{Q}_{\text {Sub }}$ and $\mathrm{F} 1_{\text {Sub }}$ both occurred at 0 degrees (self reference). d, OSI of F1 $1_{\text {Sub }}$ is plotted against OSI of QSub 
for all neurons. e, Distribution of absolute differences in preferred orientation ( $\Delta$ Pref Ori)

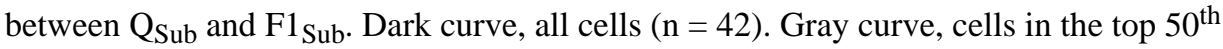
percentile of $\mathrm{F} 1_{\text {Sub }}$ OSI $(\mathrm{n}=21) . \mathbf{f}-\mathbf{g}$, Same as $(\mathbf{d}-\mathbf{e})$ for DSI and absolute differences in preferred direction ( $\Delta$ Pref Dir). Filled green markers in $\mathbf{d}$ and $\mathbf{f}$ denote the OSI and DSI values of the example cell. Data in $\mathbf{c}-\mathbf{g}$ are from $n=42$ cells from 33 mice. Error bars, mean \pm s.e.m. 

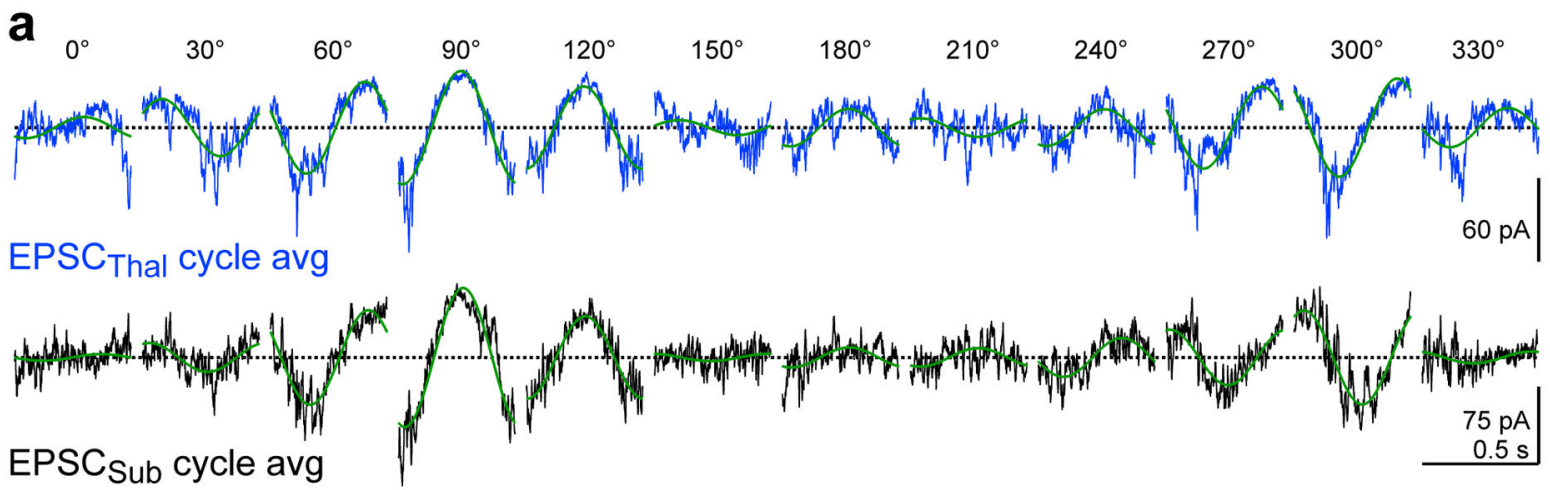

b

Example cell

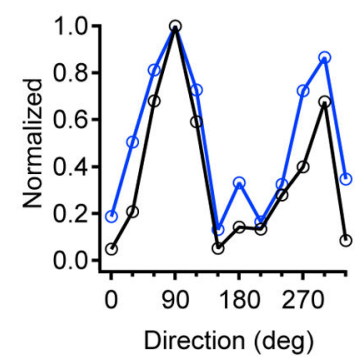

C

Population avg

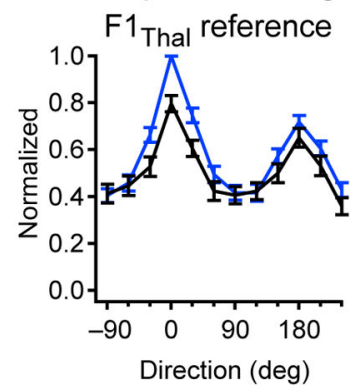

g

f
Population avg response

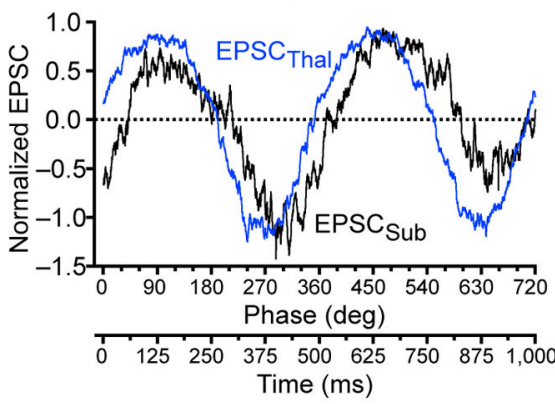

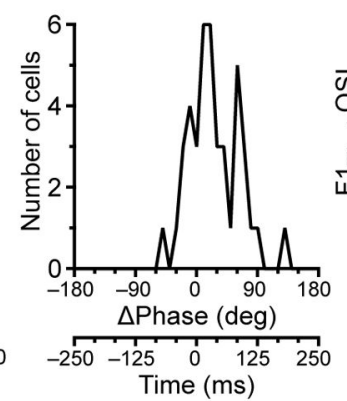

d

$\Delta$ Pref Ori

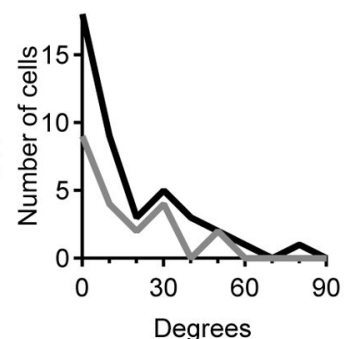

e

$\Delta$ Pref Dir

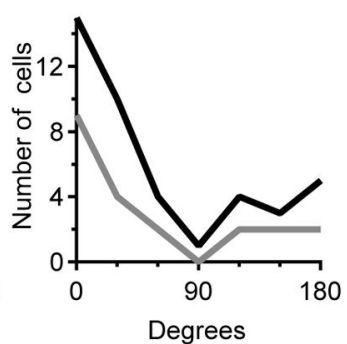

Figure 7. Co-tuning and phase relationship between thalamic and non-thalamic excitation

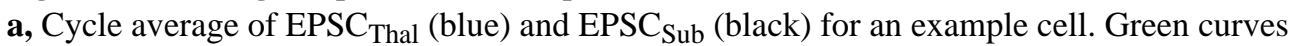
are the best-fitting sinusoids at the grating temporal frequency $(2 \mathrm{~Hz})$. b. Orientation tuning curves of $F 1_{\text {Thal }}$ (blue) and $F 1_{\text {Sub }}$ (black) for cell in a. c, Population tuning curves of $F 1_{\text {Thal }}$ (blue) and $\mathrm{F} 1_{\text {Sub }}$ (black). $\mathrm{F} 1_{\text {Thal }}$ and $\mathrm{F} 1_{\text {Sub }}$ tuning curves were aligned to the preferred direction of $F 1_{\text {Thal }}\left(\mathrm{F} 1_{\text {thal }}\right.$ reference) d, Top, distribution of absolute difference in preferred orientation ( $\Delta$ Pref Ori) between $F 1_{\text {Thal }}$ and $F 1_{\text {Sub }}$. Dark curve, all cells $(n=42)$. Gray curve, cells in the top 50th percentile of $F 1_{\text {Thal }}$ OSI $(n=21)$. Bottom, OSI of $F 1_{\text {Thal }}$ is plotted against OSI of $F 1_{\text {Sub }}$ for all neurons. e, Same as $\mathbf{d}$ for absolute differences in preferred direction ( $\triangle$ Pref Dir) and DSI. f, Population average of EPSC $_{\text {Thal }}$ (blue) and EPSC $_{S u b}$ (black) over two grating cycles at the preferred direction of $\mathrm{F}_{\mathrm{Thal}}$ and aligned to the $\mathrm{F} 1$ phase of $\mathrm{EPSC}_{\mathrm{Thal}}$. $\mathrm{g}$, Distribution of $\mathrm{F} 1$ phase difference ( $\triangle$ Phase) between $\mathrm{EPSC}_{\mathrm{Thal}}$ and 
$\mathrm{EPSC}_{\text {Sub }}$ for responses from $\mathbf{f}$. F1 phase of $\mathrm{EPSC}_{\text {Thal }}$ is set to 0 degrees. Data in $\mathbf{c}-\mathbf{g}$ are from $n=42$ cells from 33 mice. Error bars, mean \pm s.e.m. 